\title{
Generalization of Hyperbolic Perturbation Solution for Heteroclinic Orbits of Strongly Nonlinear Self-Excited Oscillator
}

\author{
Yang-Yang Chen ${ }^{1}$, Le-Wei Yan ${ }^{2}$, Ray Kai-Leung Su ${ }^{3}$, Bo Liu ${ }^{1}$ \\ 1. Key Laboratory of Vibration Control and Structural Safety, Guangzhou University, Guangzhou, 510405, P.R. China. \\ 2. Department of Engineering Mechanics, Guangzhou University, Guangzhou, 510405, P.R. China. \\ 3. Department of Civil Engineering, The University of Hong Kong, Pokfulam Road, Hong Kong, P.R. China
}

\begin{abstract}
A generalized hyperbolic perturbation method for heteroclinic solutions is presented for strongly nonlinear self-excited oscillators in the more general form of $\ddot{x}+g(x)=\varepsilon f(\mu, x, \dot{x})$. The advantage of this work is that heteroclinic solutions for more complicated and strong nonlinearities can be analytically derived, and the previous hyperbolic perturbation solutions (Chen and Chen, 2009) for Duffing type oscillator can be just regarded as a special case of the present method. The applications to cases with quadratic-cubic nonlinearities and with quintic-septic nonlinearities are presented. Comparisons with other methods are performed to assess the effectiveness of the present method.
\end{abstract}

\section{Keywords}

Generalized hyperbolic function, hyperbolic perturbation method, heteroclinic orbits, heteroclinic bifurcation

\section{Introduction}

Homoclinic and heteroclinic orbits have been widely studied in nonlinear dynamics problems such as global bifurcation, chaotic prediction, and soliton control problems, et al (Nayfeh and Balachandran, 1995; Guckenheimer and Holmes, 2002; Nayfeh and Pai, 2004). For example, the threshold for the onset of chaos in asymmetric non-conservative nonlinear dynamic systems can be considered by an occurrence of homoclinic or heteroclinic bifurcation (Feng et al., 2012). Some optical 
soliton pulse control analysis call for analytically constructing the homoclinic or heteroclinic solution shapes of self-excited oscillators (Uzunov, 2010; Uzunov and Arabadzhiev, 2011). Particularly, in many cases of self-excited systems, heteroclinic connections can be regarded as the breaks or generations of limit cycles, and a heteroclinic orbit can be regarded as the maximum vibration amplitude boundary of a corresponding limit cycle motion under parameter control. Such a typical phenomenon can be illustrated more clearly by an example in the Appendix.

Although homoclinic and heteroclinic connections become more complicated as a conservative system is perturbed into a non-conservative system, Melnikov (Melnikov, 1963) has presented a classical global analysis method, which has been widely applied to derive conditions on existence of homoclinic or heteroclinic connections (Nayfeh and Balachandran, 1995; Guckenheimer and Holmes, 2002). A heteroclinic connection, namely, a heteroclinic bifurcation, is said to have occurred if a heteroclinic orbit is created or destroyed as a control parameter is varied. The Melnikov criterion can help in an analytical way to ascertain the values of different parameters for which heteroclinic bifurcations occur. As a typical category of non-conservative systems, the self-excited oscillator can be expressed by the equation as below,

$$
\ddot{x}+g(x)=\varepsilon f(\mu, x, \dot{x}),
$$

where the restoration force term $g(x)$, and the self-excited force and damping term $f(\mu, x, \dot{x})$ are arbitrary polynomial nonlinear functions of their arguments. Here $\mu$ is considered as the bifurcation control parameter. Many systems in the form of Eq. (1) have been investigated by the Melnikov method in classical works (Nayfeh and Balachandran, 1995; Guckenheimer and Holmes, 2002). The basic mechanism of the Melnikov method to determine a heteroclinic connection of a self-excited system, can be typically illustrated in Fig.1, in which two saddle points, labeled by $\mathrm{P}_{1}$ and $\mathrm{P}_{2}$, possess their stable manifolds labeled by $\Gamma_{11}, \Gamma_{13}, \Gamma_{22}$, and $\Gamma_{24}$, and their unstable manifolds labeled by $\Gamma_{12}, \Gamma_{13}$, $\Gamma_{21}$, and $\Gamma_{23}$. The Melnikov method gives an analytical measurement of the distance between such stable and unstable manifolds, by setting a local cross-section $\sum$ to cut the manifolds at an arbitrary time position, with the intersection points at $\zeta^{\delta}$ and $\zeta^{\mathrm{u}}$ in the local coordinate. To yield the heteroclinic orbit, namely, to obtain the complete intersections of the stable and unstable manifolds at the whole time domain, the so-called Melnikov criterion, can be derived to control the distance of intersection points in $\sum$ become zero, i.e. $\zeta^{\mathrm{u}}-\zeta^{\mathrm{s}}=0$. After the classical development history of the Melnikov method, 
improved or novel techniques in higher efficiency were still being studied to investigate the occurrences of homoclinic or heteroclinic orbits (Belhaq and Lakrad, 2000; Belhaq et al., 2000; Lenci and Rega, 2003; Cao et al., 2006; Rega and Lenci, 2008; Zhang et al., 2008).

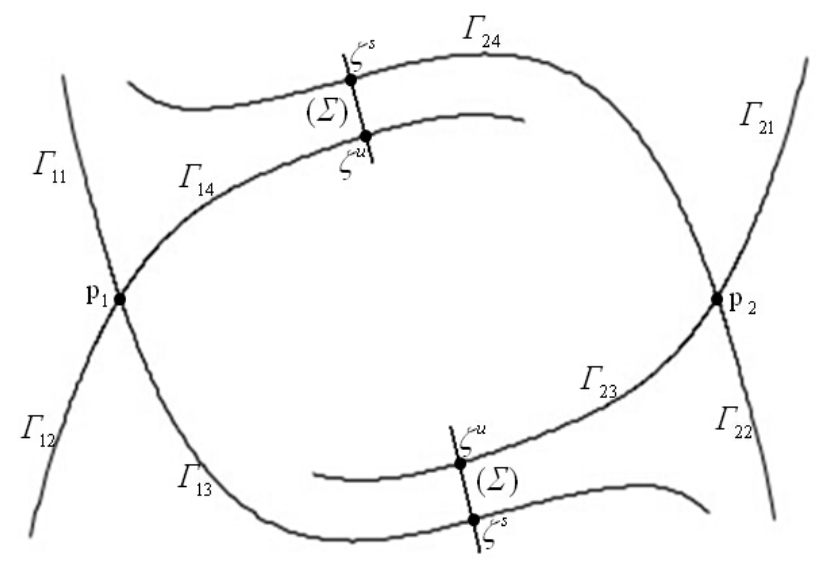

Figure 1. Distance measurement of typical stable and unstable manifolds by Melnikov method

Furthermore, in the past twenty years, many researchers presented their novel works not only for determining homoclinic or heteroclinic bifurcations, but also for constructing solutions of homoclinic or heteroclinic orbits of strongly nonlinear self-excited oscillators. These works can be briefly summarized but are not limited to: the derivation of exact solutions for the some special systems (Hale et al., 2000), the perturbation methods based on trigonometric functions and nonlinear time transformation (Li et al., 2013), the perturbation-incremental methods based on trigonometric functions and nonlinear time transformation (Xu et al., 1996; Chan et al., 1997; Cao et al., 2011), and the perturbation methods based on hyperbolic functions with Lindstedt-Poincaré procedure (Chen et al., 2009; Chen et al., 2010) or nonlinear time transformations (Chen and Chen, 2009; Chen et al., 2012), et al. On the other hand, the methods for constructing homoclinic and heteroclinic orbits of both autonomous and non-autonomous systems, have also been developed based on Padé and quasi-Padé approximants (Mikhlin and Manucharyan, 2003; Manucharyan and Mikhlin, 2005).

Nevertheless, to the best of the authors' knowledge, there still remains desirable development in this research area, as disadvantages can be found as followed. Firstly, many analytical methods (Mikhlin and Manucharyan, 2003; Manucharyan and Mikhlin, 2005; Chen and Chen, 2009; Chen et al., 2009; Chen et al., 2010) for strongly autonomous systems are only focused on the simplest nonlinear systems: the Duffing type oscillator (single cubic nonlinearity) or the Helmholtz type oscillator (single quadratic nonlinearity), as for more complicated nonlinearities an analytical homoclinic or heteroclinic 
solution will often become more difficult to be achieved. Secondly, many efficient methods (Xu et al., 1996; Chan et al., 1997; Chen and Chen, 2009; Cao et al., 2011; Li et al., 2013) for strongly nonlinear oscillators can only solved the homoclinic or heteroclinic solutions under their defined nonlinear time scales. That means, the solutions are yet implicit in respect to the original time $t$, and thus they are abstract and cumbersome to be handled in practical applications. For example, to investigate a theoretical model of a soliton shape propagating in an optical fiber, demands such an explicit homoclinic or heteroclinic solution of a corresponding oscillatory system (Uzunov, 2010; Uzunov and Arabadzhiev, 2011).

In this paper, a generalized hyperbolic perturbation procedure for heteroclinic solutions will be proposed for strongly nonlinear self-excited oscillators in the more general form of Eq. (1). By the method, heteroclinic solutions for more complicated and polynomial strong nonlinearities can be analytically derived, and the author's previous method (Chen and Chen, 2009) can be grouped into a special case of the present method. Improved procedures to seek new explicit perturbation solutions are also included. The applications to cases with quadratic-cubic nonlinearities and with quintic-septic nonlinearities will also be presented, in which the comparisons with other the typical methods are also given.

\section{Generating heteroclinic solutions by generalized hyperbolic functions}

Consider the conservative generating system of Eq. (1), i.e.

$$
\ddot{x}+g(x)=0 \text {. }
$$

The time integral of the equation is

$$
\frac{1}{2} \dot{x}^{2}+V(x)=E
$$

in which

$$
V(x)=\int_{0}^{x} g(u) \mathrm{d} u
$$

Here, $\dot{x}^{2} / 2$ and $V(x)$ can be regarded as the kinetic and the potential energies of the oscillatory system, respectively, and $E$ is the integration constant which can be interpreted as the total mechanical energy. Typical potential energy curve and phase portrait for a heteroclinic solution of Eq. (2) can be shown in Fig.2, in which a pair of so-called heteroclinic half-orbits, $\Lambda_{1}$ and $\Lambda_{2}$, is formed under the following conditions: 
(i) On the potential energy curve, there exists two maximal points $A\left(-a_{0}+b, V\left(-a_{0}+b\right)\right)$ and $B\left(a_{0}+b, V\left(a_{0}+b\right)\right)$ corresponding to the saddle points on the phase portrait, i.e.,

$$
g\left(-a_{0}+b\right)=g\left(a_{0}+b\right)=0, \quad g^{\prime}\left(-a_{0}+b\right) \leq 0, \quad g^{\prime}\left(a_{0}+b\right) \leq 0,
$$

(ii) On the potential energy curve, all points between $A$ and $B$ are at lower altitude than $A$ or $B$, i.e.

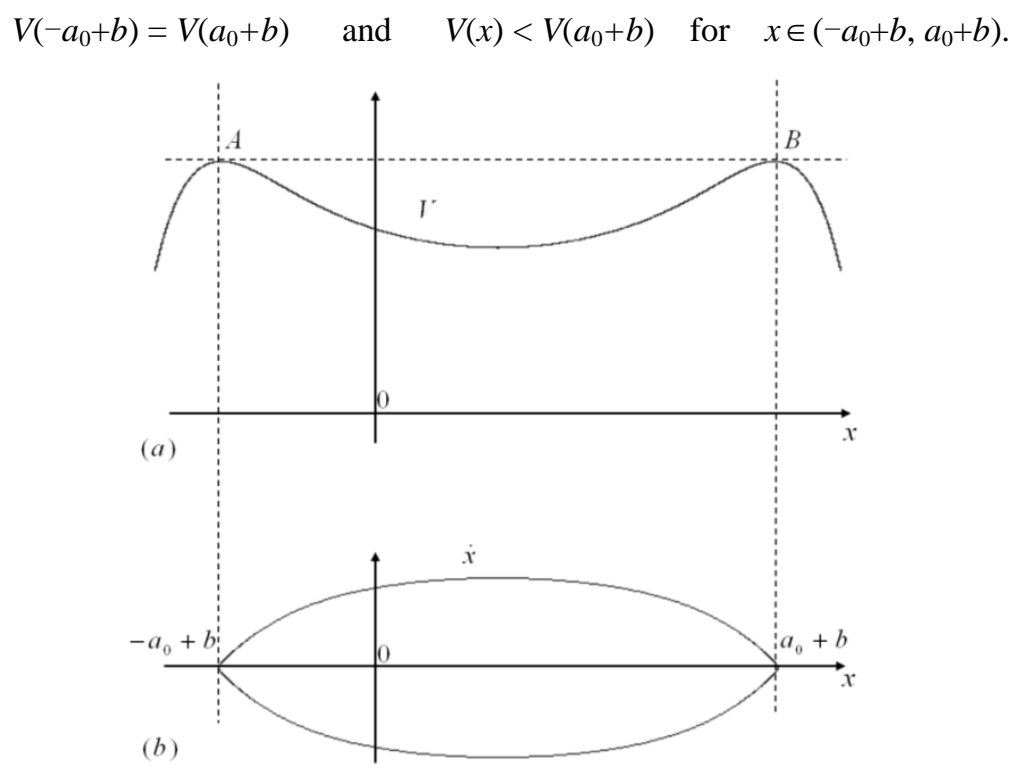

Figure 2. Typical ( $a$ ) potential energy curve and $(b)$ phase portrait of a generating heteroclinic solution

According to classical qualitative theory, here the heteroclinic solutions satisfies the so-called heteroclinic condition described as follow: As time $t \rightarrow+\infty$, a phase point $\left(x_{0}, \dot{x}_{0}\right)$ on $\Lambda_{1}$ approaches saddle point $B$, and a phase point $\left(x_{0}, \dot{x}_{0}\right)$ on $\Lambda_{2}$ approaches saddle point $A$, respectively. While as time $t \rightarrow-\infty$, a phase point $\left(x_{0}, \dot{x}_{0}\right)$ on $\Lambda_{1}$ approaches saddle point $A$ and a phase point $\left(x_{0}, \dot{x}_{0}\right)$ on $\Lambda_{2}$ approaches saddle point $B$, respectively.

To construct the heteroclinic solution of Eq. (2), one can firstly recall the typical case of $g(x)=$ $c_{1} x+c_{3} x^{3}$ (Chen and Chen, 2009), for which the heteroclinic solution can be expressed as

$$
x_{0}=a_{0} \tanh \omega_{0} t
$$

where $a_{0}$ and $\omega_{0}$ are constant. To facilitate the subsequent formulation, Eq. (7) can be rewritten as

$$
x_{0}=a_{0} \tanh \tau,
$$

and

$$
\frac{d \tau}{d t}=\omega_{0}
$$

in which $\omega_{0}$ is a constant. As 


$$
\operatorname{sech}( \pm \infty)=\tanh (0)=0, \operatorname{sech}(0)=1, \tanh ( \pm \infty)= \pm 1
$$

it is trivial that solution governed by Eq. (8) satisfies the heteroclinic condition with $\left(-a_{0}, 0\right)$ and $\left(a_{0}, 0\right)$ being the heteroclinic points.

For those more general cases in which $g(x) \neq c_{1} x+c_{3} x^{3}$ and the heteroclinic points are not limited to $\left(-a_{0}, 0\right)$ and $\left(a_{0}, 0\right)$, one can assume the heteroclinic solution in a similar but more general form, i.e.

$$
x_{0}=a_{0} \tanh \tau+b
$$

Here the constants $a_{0}$ and $b$, which control the position of heteroclinic points $\left(-a_{0}+b, 0\right)$ and $\left(a_{0}+b, 0\right)$, can be determined by Eqs. (5) and (6). Furthermore, noting that Eq. (9) can be regarded as a linear time transformation from $t$ to $\tau$, one can also introduce a more general time transformation from $t$ to $\tau$ for solution governed by Eq. (11), i.e.

$$
\frac{d \tau}{d t}=\omega_{0}(\tau)
$$

where $\omega_{0}(\tau)$ is not limited to a constant, but can be a bounded function for all $\tau$. Then

$$
\dot{x}_{0}=a_{0} \omega_{0}(\tau) \operatorname{sech}^{2} \tau
$$

As the total mechanical energy of the heteroclinic motion is

$$
E_{0}=V\left(-a_{0}+b\right)=V\left(a_{0}+b\right)
$$

one has

$$
\frac{1}{2} \omega_{0}^{2} x_{0}^{\prime 2}+V\left(x_{0}\right)=V\left(a_{0}+b\right)
$$

in which $x_{0}^{\prime}$ denotes the first derivative of $x_{0}$ with respect to $\tau$. From Eq. (15), $\omega_{0}(\tau)$ can be determined by

$$
\omega_{0}(\tau)=\frac{1}{x_{0}^{\prime}} \sqrt{2\left[V\left(a_{0}+b\right)-V\left(x_{0}\right)\right]} .
$$

Therefore, the solution for heteroclinic orbits of Eq. (2) can be expressed by Eqs. (11), (13) and (16), in which the functions $\operatorname{sech} \tau(t)$ and $\tanh \tau(t)$ will be employed as the basic functions in the following perturbation procedures and can be regarded as the generalized hyperbolic functions for heteroclinic orbits.

\section{Generalized hyperbolic perturbation method for heteroclinic solutions}

If the self-excited force and damping term $\varepsilon f(\mu, x, \dot{x})$ in Eq. (1) becomes nonzero, the conservative phase portrait structure will be destroyed, however, the saddle points $\left(-a_{0}+b, 0\right)$ and $\left(a_{0}+b\right.$, 
0) remain the saddles during the parameter control of the self-excited system (Nayfeh and Balachandran, 1995; Guckenheimer and Holmes, 2002) (see Appendix as an illustration). Therefore, based on the generated heteroclinic solutions governed by Eq. (11), one can start the generalized perturbation procedure by expanding the heteroclinic solution of Eq. (1) in a series form as

$$
x=x_{0}+\varepsilon x_{1}+\cdots+\varepsilon^{n} x_{n}+\cdots, \quad(n=0,1, \ldots),
$$

where for $n \geqslant 1$,

$$
x_{n}=a_{n} \tanh \tau \operatorname{sech} \tau,
$$

and

$$
x_{n}^{\prime}=a_{n} \operatorname{sech} \tau\left(2 \operatorname{sech}^{2} \tau-1\right) .
$$

The higher order solutions in the form of Eqs. (18) and (19) can ensure the heteroclinic solution expressed by Eq. (17) satisfy the heteroclinic condition with the saddle points at $\left(-a_{0}+b, 0\right)$ and $\left(a_{0}+b\right.$, 0 ). Furthermore, the time transformation from $t$ to $\tau$ is also expanded in respect to the perturbation parameter $\varepsilon$ by the equation

$$
\frac{d \tau}{d t}=\omega(\tau)=\omega_{0}(\tau)+\varepsilon \omega_{1}(\tau)+\cdots+\varepsilon^{n} \omega_{n}(\tau)+\cdots,
$$

in which $\omega_{n}(\tau)$ are assumed as bounded nonlinear time functions to be determined by subsequent procedures and for $n \geqslant 1$,

$$
\omega_{n}(0)=0 .
$$

Substituting Eqs. (17) and (20) into Eq. (1) and equating coefficients of like powers of $\varepsilon$ yields the following equations:

$$
\begin{aligned}
& \varepsilon^{0}: \omega_{0} \frac{d}{d \tau}\left(\omega_{0} x_{0}^{\prime}\right)+g\left(x_{0}\right)=0, \\
& \varepsilon^{1}: \omega_{0} \frac{d}{d \tau}\left(\omega_{1} x_{0}^{\prime}\right)+\omega_{1} \frac{d}{d \tau}\left(\omega_{0} x_{0}^{\prime}\right)+\omega_{0} \frac{d}{d \tau}\left(\omega_{0} x_{1}^{\prime}\right)+g,_{x}\left(x_{0}\right) x_{1}=f\left(\mu, x_{0}, \omega_{0} x_{0}^{\prime}\right), \\
& \varepsilon^{2}: \quad \omega_{0} \frac{d}{d \tau}\left(\omega_{2} x_{0}^{\prime}\right)+\omega_{2} \frac{d}{d \tau}\left(\omega_{0} x_{0}^{\prime}\right)+\omega_{0} \frac{d}{d \tau}\left(\omega_{0} x_{2}^{\prime}\right)+\omega_{1} \frac{d}{d \tau}\left(\omega_{0} x_{1}^{\prime}\right)+\omega_{0} \frac{d}{d \tau}\left(\omega_{1} x_{1}^{\prime}\right) \\
&+\omega_{1} \frac{d}{d \tau}\left(\omega_{1} x_{0}^{\prime}\right)+g,_{x}\left(x_{0}\right) x_{2}+\frac{1}{2} g,_{x x}\left(x_{0}\right) x_{1}^{2} \\
&=f,,_{x}\left(\mu, x_{0}, \omega_{0} x_{0}^{\prime}\right) x_{1}+f,,_{\dot{x}}\left(\mu, x_{0}, \omega_{0} x_{0}^{\prime}\right)\left(\omega_{0} x_{1}^{\prime}+\omega_{1} x_{0}^{\prime}\right),
\end{aligned}
$$


where $g,_{x}=\partial g / \partial x, g,_{x x}=\partial^{2} g / \partial x^{2}$, etc. Thus one can solve the above linear equations one by one, and determine each order for solutions $x_{0}, x_{1}, x_{2} \ldots$

It can be seen that Eq. (22) is obtained from Eq. (2) via the transformation by Eq. (12). Hence, the heteroclinic solution of Eq. (22) can be expressed by Eq. (8). By multiplying both sides of Eq. (23) by $x_{0}^{\prime}$ and integrating it from $\tau_{0}$ to $\tau$, one obtains

$$
\left.\omega_{0} \omega_{1} x_{0}^{\prime 2}\right|_{\tau_{0}} ^{\tau}=\int_{\tau_{0}}^{\tau} x_{0}^{\prime} f\left(\mu, x_{0}, \omega_{0} x_{0}^{\prime}\right) d \tau-\left.\omega_{0}^{2} x_{0}^{\prime} x_{1}^{\prime}\right|_{\tau_{0}} ^{\tau}-\left.x_{1} g\left(x_{0}\right)\right|_{\tau_{0}} ^{\tau},
$$

or

$$
\left.\omega_{0} \omega_{1} x_{0}^{\prime 2}\right|_{\tau_{0}} ^{\tau}=I(\tau)-I\left(\tau_{0}\right)-\left.\omega_{0}^{2} x_{0}^{\prime} x_{1}^{\prime}\right|_{\tau_{0}} ^{\tau}-\left.x_{1} g\left(x_{0}\right)\right|_{\tau_{0}} ^{\tau}
$$

where

$$
I(\tau)=\int_{0}^{\tau} x_{0}^{\prime} f\left(\mu, x_{0}, \omega_{0} x_{0}^{\prime}\right) d \tau=\int_{0}^{\tau} x_{0}^{\prime} f\left(\mu, x_{0}, \sqrt{2\left[V\left(a_{0}+b\right)-V\left(x_{0}\right)\right]}\right) d \tau .
$$

Recalling Eq. (10), one has

$$
\begin{gathered}
x_{0}(0)=b, \quad x_{0}^{\prime}(0)=a_{0}, \quad x_{0}( \pm \infty)= \pm a_{0}+b, \quad x_{n}^{\prime}(0)= \pm a_{n}, \\
x_{0}^{\prime}( \pm \infty)=x_{n}(0)=x_{n}( \pm \infty)=x_{n}^{\prime}( \pm \infty)=0
\end{gathered}
$$

Therefore, letting $\tau_{0}=-\infty, \tau=+\infty$ and $\tau_{0}=0$ in turn, and noting the conditions governed by Eqs. (5), (21) and (28), one derives three equations as follows:

$$
\begin{gathered}
I(+\infty)-I(-\infty)=0, \\
a_{1}=-\frac{I(+\infty)}{\omega_{0}^{2}(0) a_{0}}, \\
\omega_{1}=\frac{1}{\omega_{0} x_{0}^{\prime 2}}\left[I(\tau)-\omega_{0}^{2} x_{0}^{\prime} x_{1}^{\prime}+\omega_{0}^{2}(0) a_{0} a_{1}-x_{1} g\left(x_{0}\right)\right] .
\end{gathered}
$$

It can be seen that Eqs. (27) and (29), by which the heteroclinic bifurcation value, $\mu=\mu_{\mathrm{c}}$ can be determined, agree with the Melnikov criterion (Nayfeh and Balachandran, 1995; Guckenheimer and Holmes, 2002). Similar equivalent formulas can also be derived in some works (Chan et al., 1997; Zhang et al., 2008; Cao et al., 2011; Chen et al., 2010).

Then, one can determine $a_{1}, \omega_{1}$ from Eqs. (30) and (31), and obtain the heteroclinic solution as

$$
\begin{gathered}
x=a_{0} \tanh \tau+b+O\left(\varepsilon^{2}\right), \\
\dot{x}=a_{0}\left[\omega_{0}+\varepsilon \omega_{1}(\tau)\right] \operatorname{sech}^{2} \tau+O\left(\varepsilon^{2}\right) .
\end{gathered}
$$


The next order solution can be determined by a similar procedure. However, the perturbation procedure will be increasingly cumbersome as the order goes up. More importantly, the computational results will show that the solution up to the order $\varepsilon x_{1}$ is fairly accurate even for the moderately large parameter $\varepsilon$.

It is worth pointing out that the solution, governed by Eqs. (20), (32), and (33), is expressed at the nonlinear time scale $\tau$. In other words, they are yet implicit solutions in respect to the original time $t$. Although such implicit solutions can give quite accurate orbits in phase portraits for theoretical studies, they are usually much less practical for application compared to those explicit solutions in respect to the original time $t$. Unfortunately, it is also much more difficult to derive the explicit solutions that, many perturbation methods (Xu et al., 1996; Chan et al., 1997; Chen and Chen, 2009; Cao et al., 2011; Li et al., 2013) for strongly nonlinear oscillators, cannot give their solutions explicitly in respect to the original time $t$, but only presented their solutions in their nonlinear time scales.

Nevertheless, it can be found that for some important systems such as the mix-parity Helmholtz-Duffing oscillators, which will be studied in detail in Section $4, \omega_{0}$ can be derived as constants by Equation (16), i.e.

$$
\tau=\omega_{0} t
$$

For this kind of systems, Eq. (34) means the time transformation for the perturbation order of $\varepsilon^{0}$, is linear and explicit. Thus, one can introduce an approximation for Eq. (20) by substituting Eq. (34) into Eq. (20), and thus obtain an new time integration as

$$
\tau=\omega_{0} t+\varepsilon \int_{0}^{t} \omega_{1}\left(\omega_{0} t\right) d t+O\left(\varepsilon^{2}\right)
$$

Therefore, the explicit solution of heteroclinic solution can be finally expressed by Eqs. (32), (33) and (35).

It can be seen that while $g(x)=c_{1} x+c_{3} x^{3}$, the present procedures can be easily reduced to those procedures (Chen and Chen, 2009) for heteroclinic solutions of simple Duffing type oscillator. Therefore, the method can be regarded as a special case of the present method in this paper.

\section{Application to strongly quadratic-cubic nonlinearities}

\subsection{The perturbation procedure}

As an application of the present method, the following system is studied:

$$
\ddot{x}+c_{1} x+c_{2} x^{2}+c_{3} x^{3}=\varepsilon\left(\mu+\mu_{1} x\right) \dot{x}
$$


In other words,

$$
\begin{aligned}
& g\left(x_{0}\right)=c_{1} x_{0}+c_{2} x_{0}^{2}+c_{3} x_{0}^{3}, \\
& V\left(x_{0}\right)=\frac{1}{2} c_{1} x_{0}^{2}+\frac{1}{3} c_{2} x_{0}^{3}+\frac{1}{4} c_{3} x_{0}^{4}, \\
& f\left(\mu, x_{0}, \omega_{0} x_{0}^{\prime}\right)=\omega_{0} x_{0}^{\prime}\left(\mu+\mu_{1} x_{0}\right),
\end{aligned}
$$

in which $\mu_{1}$ is constant. By substituting Eqs. (37) and (38) into Eqs. (5) and (6), respectively, one has

$$
\begin{gathered}
c_{1} b+c_{2} b^{2}+c_{3} b^{3}=0, c_{1}+2 c_{2} b+3 c_{3} b^{2} \leq 0 \\
\frac{1}{2} c_{1}\left(-a_{0}+b\right)^{2}+\frac{1}{3} c_{2}\left(-a_{0}+b\right)^{3}+\frac{1}{4} c_{3}\left(-a_{0}+b\right)^{4}=\frac{1}{2} c_{1}\left(a_{0}+b\right)^{2}+\frac{1}{3} c_{2}\left(a_{0}+b\right)^{3}+\frac{1}{4} c_{3}\left(a_{0}+b\right)^{4},
\end{gathered}
$$

by which $a_{0}$ and $b$ can be determined. Eq. (27) can be rewritten as

$$
\begin{gathered}
I(\tau)=\int_{0}^{\tau} x_{0}^{\prime} f\left(\mu, x_{0}, \sqrt{2\left[V\left(a_{0}+b\right)-V\left(x_{0}\right)\right]}\right) d \tau \\
=\sqrt{2} a_{0} \int_{0}^{\tau} \operatorname{sech}^{2} \tau\left[\mu+\mu_{1}\left(a_{0} \tanh \tau+b\right)\right] \sqrt{\left[V\left(a_{0}+b\right)-V\left(a_{0} \tanh \tau+b\right)\right]} d \tau
\end{gathered}
$$

Thus substituting Eqs. (38) and (41) into Eqs. (29), (30) and (31), one can complete the calculation of the perturbation solution governed by Eqs. (32), (33) and (35).

\subsection{Examples}

Three examples are presented in this section for assessment of the present method. Cao's perturbation method (Cao et al., 2011) and Chen’s hyperbolic Lindstedt-Poincaré method (Chen et al., 2010) are also applied for the examples. As Chen's method is only available for Duffing-type oscillator, it is fail to be performed in Examples 2 and 3. Comparisons for different methods are shown in the Figures in each example.

It should also be mentioned here that, a significant advantage of the incremental-perturbation method (Xu et al., 1996; Chan et al., 1997; Cao et al., 2011) is that it can be combined with the incremental harmonic balance method to get semi-numerical and semi-analytical solutions, by which strongly nonlinear system even with arbitrary large $\varepsilon$ can be solved. However, in these results by incremental techniques, the bifurcation value of the control parameter, as well as important coefficients in solutions, are yet derived numerically. In this paper as the study is focused on purely analytical methods, the incremental part of Cao’s method will be ignored. Furthermore, as Cao’s method can only give implicit solution in respect to time $t$, it is fail to directly derive all the time history diagrams in the following examples by the method. 
Example 1. Consider the following oscillator:

$$
\ddot{x}+2 x-x^{3}=\varepsilon\left(\mu-x^{2}+x^{4}\right) \dot{x},
$$

which is a case of Eq. (36) with $c_{1}=2, c_{2}=0, c_{3}=-1, \mu_{1}=0, \mu_{2}=-1, \mu_{4}=1$. From Eq. (40), $a_{0}= \pm \sqrt{2}$ and $b=0$. From Eq. (16), $\omega_{0}=1$. By incorporating Eqs. (38) and (41), one can complete the integral calculation and get

$$
\begin{gathered}
I(\tau)=\omega_{0} a_{0}^{2} \tanh \tau\left[\left(\frac{-1}{35} \mu_{4} a_{0}^{4}-\frac{1}{15} \mu_{1} a_{0}^{2}+\frac{1}{3} \mu\right)\left(2+\operatorname{sech}^{2} \tau\right)+\left(\frac{8}{35} \mu_{4} a_{0}^{4}+\frac{1}{5} \mu_{1} a_{0}^{2}\right) \operatorname{sech}^{4} \tau-\frac{1}{7} \mu_{4} a_{0}^{4} \operatorname{sech}^{6} \tau\right] \\
=2 \tanh \tau\left[(-0.0190+0.3333 \mu)-0.5143 \operatorname{sech}^{4} \tau+0.5714 \operatorname{sech}^{6} \tau\right]
\end{gathered}
$$

Substituting Eq. (56) into Eq. (29) yields the heteroclinic bifurcation value

$$
\mu_{\mathrm{c}}=\frac{3}{35} \mu_{4} a_{0}^{4}+\frac{1}{5} \mu_{1} a_{0}^{2}=\frac{2}{35}=0.0571 .
$$

By substituting Eqs. (56) and (57) into Eq. (30), one obtains

$$
a_{1}=0
$$

By substituting Eqs. (56), (57) and (58) into Eq. (31), one obtains

$$
\omega_{1}=\frac{1}{35} a_{0}^{2} \tanh \tau\left[\mu_{4} a_{0}^{2}\left(8-5 \operatorname{sech}^{2} \tau\right)+7 \mu_{1}\right]=\left(-0.5143+0.5714 \operatorname{sech}^{2} \tau\right) \tanh \tau .
$$

Thus from Eqs. (32), (33), and (35), the heteroclinic solution can be derived as below,

$$
\begin{gathered}
x= \pm 1.4142 \tanh \tau, \\
\dot{x}= \pm 1.4142\left[1+\varepsilon\left(-0.5143+0.5714 \operatorname{sech}^{2} \tau\right) \tanh \tau\right] \operatorname{sech}^{2} \tau,
\end{gathered}
$$

where

$$
\tau=t+\varepsilon\left[0.2857+0.5143 \ln (\operatorname{sech} t)-0.2857 \operatorname{sech}^{2} t\right]
$$

To assess the efficiency of the present method, here we also solve the heteroclinic solutions of Eq. (42), by using Cao’s method and Chen’s method, respectively. The solution by Cao's method is derived as below,

$$
\begin{gathered}
x=1.4142 \cos \varphi, \\
\frac{d x}{d \varphi}=-1.4142 \sin \varphi . \\
\dot{\varphi}=\sin \varphi\left[ \pm 1+0.0514 \varepsilon \cos \varphi\left(10 \cos ^{2} \varphi-1\right)\right] .
\end{gathered}
$$

The solution by Chen's method is derived as below,

$$
x= \pm 1.4142 \tanh t-0.0808 \varepsilon\left[9 \ln (\cosh t)+5 \operatorname{sech}^{2} t\right] \operatorname{sech}^{2} t
$$


With $\varepsilon=1.2$, the phase portrait and time history diagrams of the solutions by different analytical methods and the Runge-kutta method are shown in Figs. 3-5 for comparison.

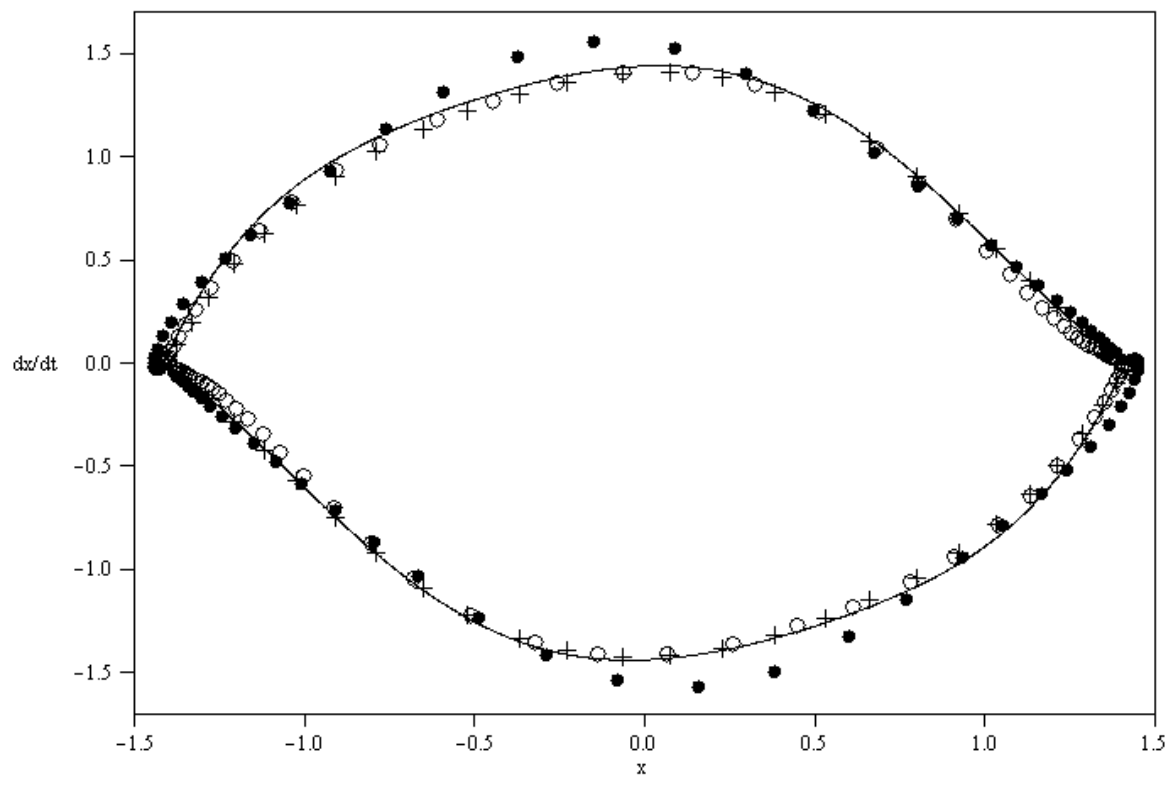

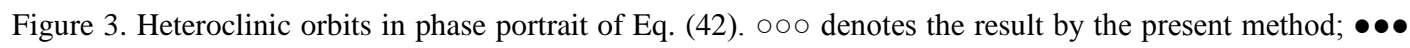
denotes the result by Chen's method; +++ denotes the result by Cao's method; - denotes the numerical orbits at $\mu_{\mathrm{c}}$ predicted by the Runge-Kutta method.

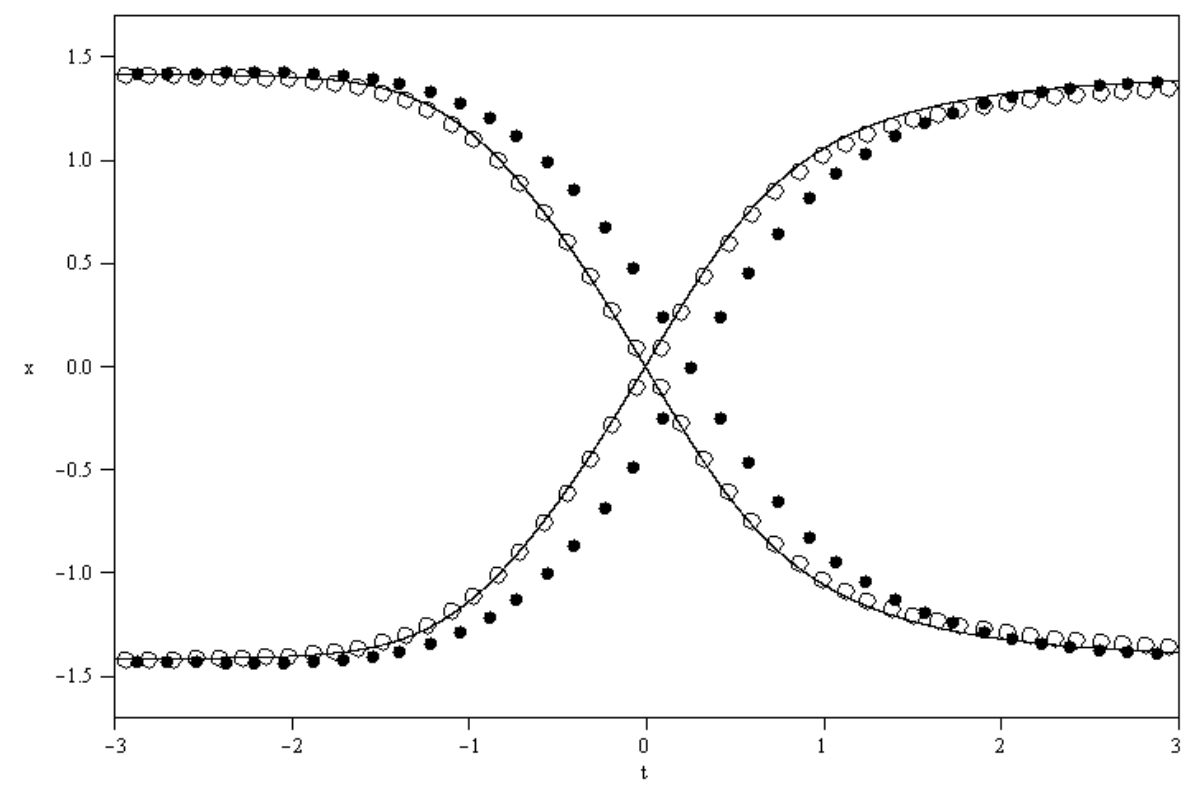

Figure 4. Amplitude history of heteroclinic solutions of Eq. (42). $\circ \circ \circ$ denotes the result by the present method; $\bullet \bullet$ denotes the result by Chen's method; - denotes the numerical result at $\mu_{\mathrm{c}}$ by the Runge-Kutta method. 



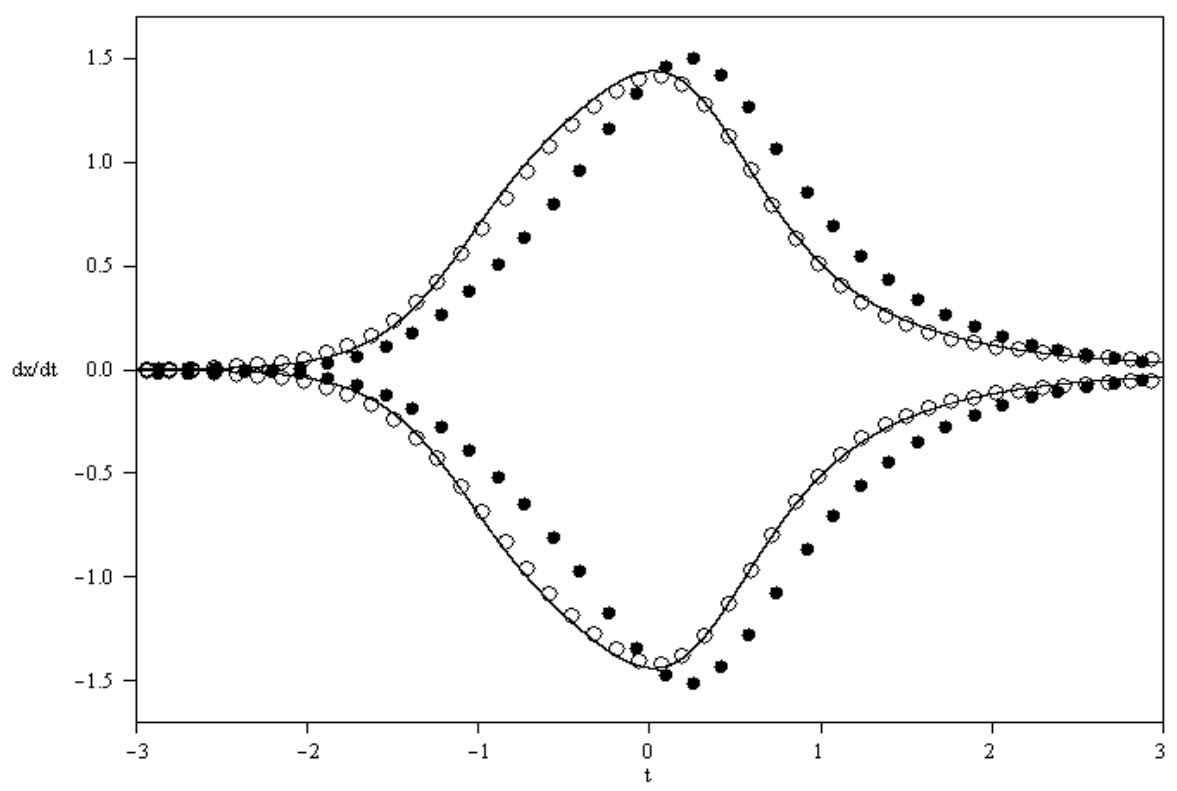

Figure 5. Velocity history of heteroclinic solutions of Eq. (42). 000 denotes the result by the present method; $\bullet \bullet \bullet$ denotes the result by Chen's method; — denotes the numerical result at $\mu_{\mathrm{c}}$ by the Runge-Kutta method.

It can be seen from Figs 3-5 that, Cao's method and the present method show better accuracy than those by Chen's hyperbolic Lindstedt-Poincaré method. That means the solutions constructed by nonlinear time transformation should be better techniques for high accuracy requirement.

It can also be seen from Eqs. (50)-(52) and Figs. 4 and 5 that, by Cao's method it is too cumbersome to solve the solution in explicit form in respect to time $t$. The reason is that with this method, the original infinite time domain $(-\infty \rightarrow+\infty)$ is transferred into a half period $(\pi \rightarrow 0)$ nonlinear time domain, where the nonlinear time scale $\varphi$ is difficult to expressed by the original time $t$, explicitly.

In this paper, the procedure of using numerical Runge-Kutta method to determine the value of parameter $\mu$ of the heteroclinic orbit follows that of Merkin and Needham (Merkin and Needham, 1986). Numerical integration is conducted for a given value of $\varepsilon$ starting from a value of $\mu$ with which there is a limit cycle. To obtain such a numerical limit cycle, the initial value is convenient to choose as any point located in its attraction basin (see Figs. 15-18 in the Appendix) will approach to the limit cycle as $t \rightarrow+\infty$ or $t \rightarrow-\infty$. Then it is repeated for increasing or reducing $\mu$ until a value of $\mu$ is reached such that the limit cycle breaks, namely the heteroclinic bifurcation occurs. Then, by successfully reducing the interval of $\mu$ within which a limit cycle is destroyed, a critical value $\mu_{c}$ can be identified such that a limit cycle can be found at $\mu=\mu_{c}$ but not at $\mu=\mu_{c} \pm \Delta$ where $\Delta$ is a small preset tolerance. 
Here, $\Delta$ is taken to be $10^{-9}$. Using this trial and error approach, $\mu_{c}=0.052765730=0.0528$ when $\varepsilon=1.2$ in Eq.(42). The value is very closed to that obtained by the present method. Particularly, it is worth noting that the limit cycle at $\mu_{c}$, which is extremely near the heteroclinic connection, can fit the shape of the exact heteroclinic orbit excellently with the ignorable errors $\Delta=10^{-9}$ of $\mu_{\mathrm{c}}$. Thus as a numerical tool for assessing the shapes of out analytical orbits, such procedures are given to made comparison with our analytical approximate solutions in all the examples of the paper.

Example 2. Consider the following oscillator:

$$
\ddot{x}-x+3 x^{2}-2 x^{3}=\varepsilon(\mu-2 x) \dot{x},
$$

which is a case of Eq. (36) with $c_{1}=-1, c_{2}=3, c_{3}=-2, \mu_{1}=-2$. From Eq. (40), $a_{0}= \pm 0.5$ and $b=0.5$. From Eq. (16), $\omega_{0}=0.5$. By incorporating Eqs. (38) and (41), one can complete the integral calculation and get

$$
\begin{aligned}
I(\tau) & =\frac{1}{12} \omega_{0} a_{0}^{2}\left[3 \mu_{1} a_{0}\left(1-\operatorname{sech}^{4} \tau\right)+4\left(\mu+\mu_{1} b\right)\left(2+\operatorname{sech}^{2} \tau\right) \tanh \tau\right] \\
& =0.0313\left(\operatorname{sech}^{4} \tau-1\right)+0.0417(\mu-1)\left(2+\operatorname{sech}^{2} \tau\right) \tanh \tau
\end{aligned}
$$

Substituting Eq. (56) into Eq. (29) yields the heteroclinic bifurcation value

$$
\mu_{\mathrm{c}}=-\mu_{1} b=1
$$

By substituting Eqs. (56) and (57) into Eq. (30), one obtains

$$
a_{1}=-\frac{\mu_{1} a_{0}^{2}}{4 \omega_{0}}=0.25
$$

By substituting Eqs. (56), (57) and (58) into Eq. (31), one obtains

$$
\begin{aligned}
\omega_{1}= & 0.25 \mu_{1}\left\{a_{0} \operatorname{sech} \tau\left(2 \operatorname{sech}^{2} \tau-\operatorname{sech} \tau-1\right)+\frac{\sinh \tau}{\omega_{0}^{2}}\left[c_{1}\left(a_{0} \tanh \tau+b\right)+c_{2}\left(a_{0} \tanh \tau+b\right)^{2}\right.\right. \\
& \left.\left.+c_{3}\left(a_{0} \tanh \tau+b\right)^{3}\right]\right\}=0.25 \operatorname{sech} \tau(\operatorname{sech} \tau-1)
\end{aligned}
$$

Thus from Eqs. (32), (33), and (35), the heteroclinic solution can be derived as below,

$$
\begin{gathered}
x=0.25 \tanh \tau( \pm 2+\varepsilon \operatorname{sech} \tau)+0.5, \\
\dot{x}=0.0625 \operatorname{sech} \tau[2+\varepsilon \operatorname{sech} \tau(\operatorname{sech} \tau-1)]\left[ \pm 2 \operatorname{sech} \tau+\varepsilon\left(2 \operatorname{sech}^{2} \tau-1\right)\right],
\end{gathered}
$$

where

$$
\tau=0.7071 t+0.2121[\arctan (\sinh (0.7071 t))-\tanh (0.7071 t)]
$$

The solution by Cao's method can be derived as below, 


$$
\begin{gathered}
x=1.4142 \cos \varphi, \\
\frac{d x}{d \varphi}=-1.4142 \sin \varphi . \\
\dot{\varphi}=\sin \varphi\left[ \pm 1+0.0514 \varepsilon \cos \varphi\left(10 \cos ^{2} \varphi-1\right)\right] .
\end{gathered}
$$

With $\varepsilon=0.4$, the phase portraits and time history diagrams of the solutions by different methods are shown in Figs. 6-8. The Runge-Kutta numerical solution at $\mu_{\mathrm{c}}$ is also shown for comparison. Here, the critical value $\mu_{\mathrm{c}}=1.0000$ is obtained, which is in agreement with that obtained by the present method.

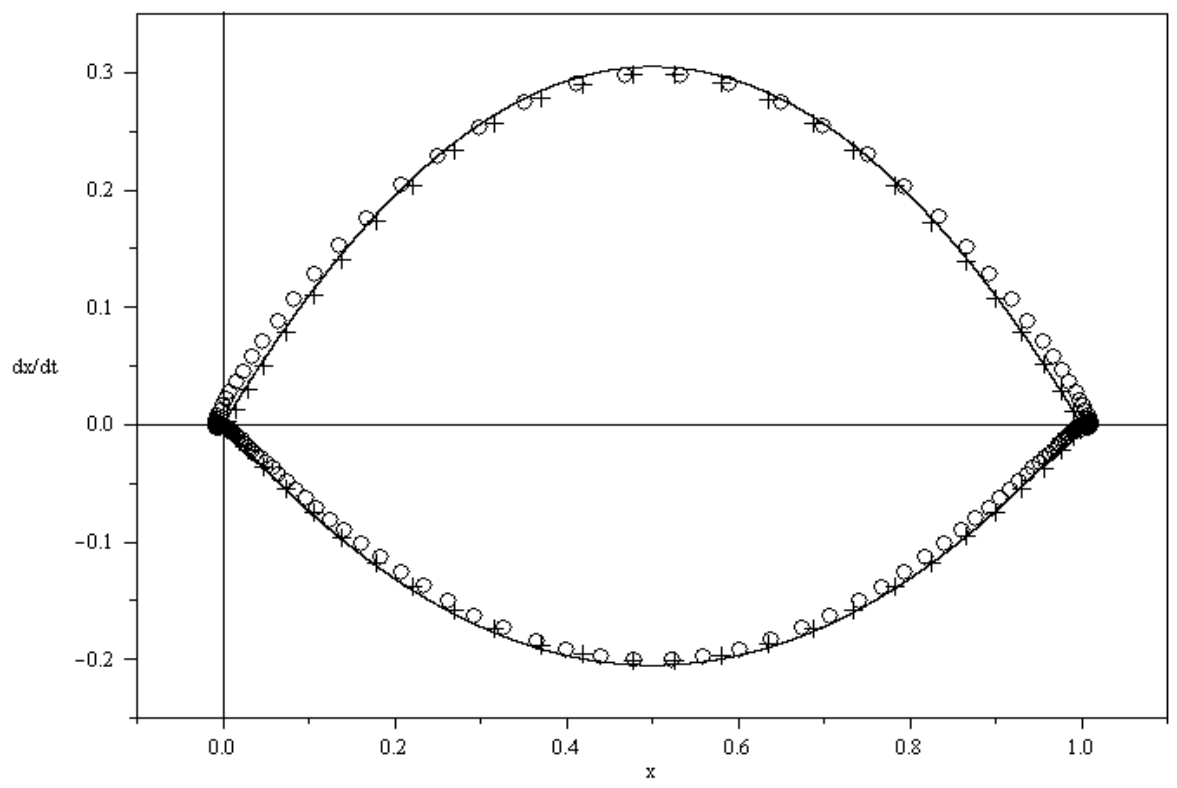

Figure 6. Heteroclinic orbits in phase portrait of Eq. (55). $\circ 00$ denotes the result by the present method; + + + denotes the result by Cao's method; - denotes the numerical orbits at $\mu_{\mathrm{c}}$ predicted by the Runge-Kutta method. 


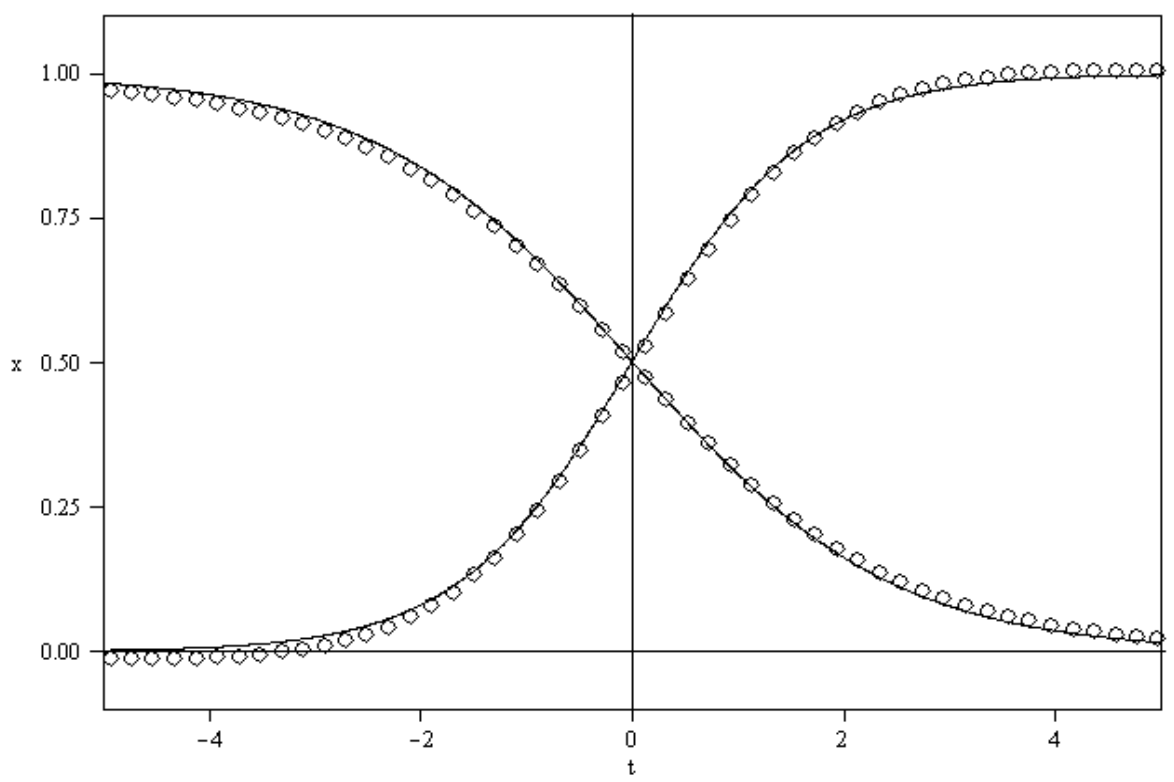

Figure 7. Amplitude history of heteroclinic solutions of Eq. (55). $० \circ \circ$ denotes the result by the present method; — denotes the numerical result at $\mu_{\mathrm{c}}$ by the Runge-Kutta method.

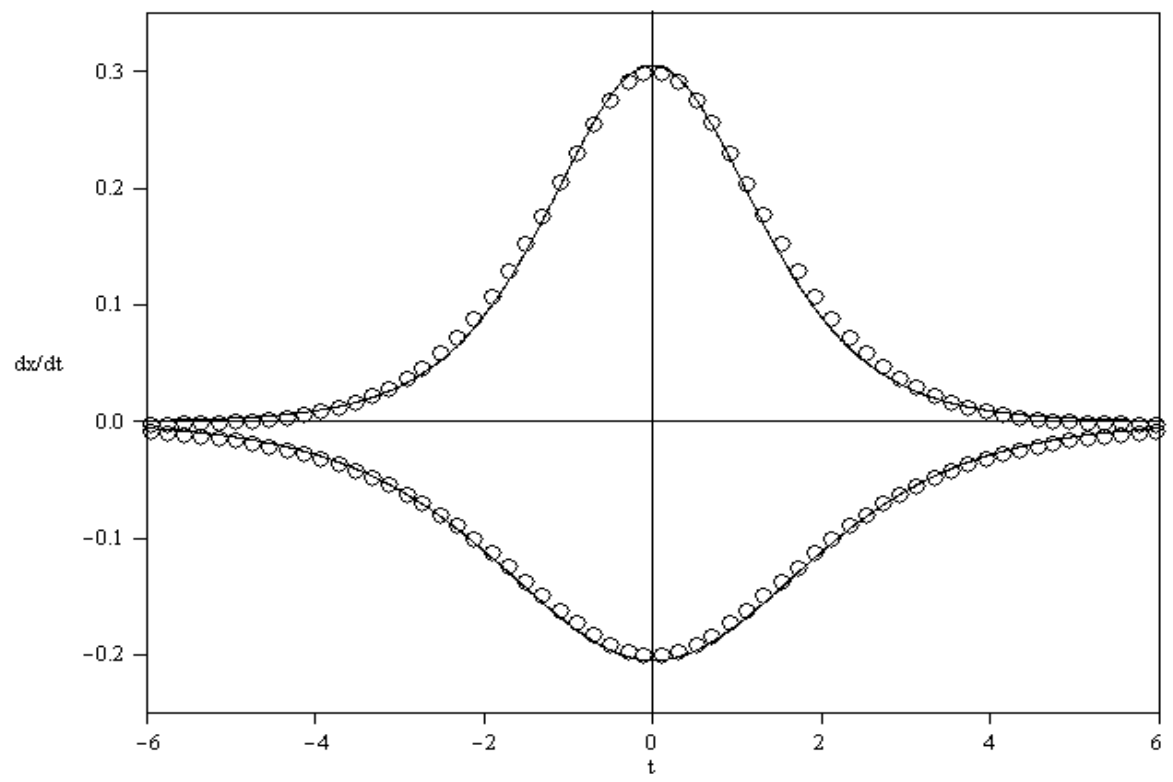

Figure 8. Velocity history of heteroclinic solutions of Eq. (55). $\circ \circ \circ$ denotes the result by the present method; — denotes the numerical result at $\mu_{\mathrm{c}}$ by the Runge-Kutta method.

Example 3. Consider the following oscillator:

$$
\ddot{x}-2 x-3 x^{2}-x^{3}=\varepsilon(\mu+0.5 x) \dot{x},
$$


which is a case of Eq. (36) with $c_{1}=-2, c_{2}=-3, c_{3}=-1$ and $\mu_{1}=0.5$. From Eq. (40), $a_{0}= \pm 1$ and $b=$ -1. From Eq. (16), $\omega_{0}=0.7071$. By incorporating Eqs. (38) and (41), one can complete the integral calculation and get

$$
\begin{aligned}
I(\tau)= & \frac{1}{12} \omega_{0} a_{0}^{2}\left[3 \mu_{1} a_{0}\left(1-\operatorname{sech}^{4} \tau\right)+4\left(\mu+\mu_{1} b\right)\left(2+\operatorname{sech}^{2} \tau\right) \tanh \tau\right] \\
& =0.0884\left(\operatorname{sech}^{4} \tau-1\right)+0.1179(2 \mu-1)\left(2+\operatorname{sech}^{2} \tau\right) \tanh \tau
\end{aligned}
$$

Substituting Eq. (56) into Eq. (29) yields the heteroclinic bifurcation value

$$
\mu_{\mathrm{c}}=-\mu_{1} b=0.5
$$

By substituting Eqs. (56) and (57) into Eq. (30), one obtains

$$
a_{1}=-\frac{\mu_{1} a_{0}^{2}}{4 \omega_{0}}=-0.1768 .
$$

By substituting Eqs. (56), (57) and (58) into Eq. (31), one obtains

$$
\begin{aligned}
\omega_{1}= & 0.25 \mu_{1}\left\{a_{0} \operatorname{sech} \tau\left(2 \operatorname{sech}^{2} \tau-\operatorname{sech} \tau-1\right)+\frac{\sinh \tau}{\omega_{0}^{2}}\left[c_{1}\left(a_{0} \tanh \tau+b\right)+c_{2}\left(a_{0} \tanh \tau+b\right)^{2}\right.\right. \\
& \left.\left.+c_{3}\left(a_{0} \tanh \tau+b\right)^{3}\right]\right\}=0.125 \operatorname{sech} \tau(1-\operatorname{sech} \tau) .
\end{aligned}
$$

Thus from Eqs. (32), (33), and (35), the heteroclinic solution can be derived as below,

$$
\begin{gathered}
x=\tanh \tau( \pm 1-0.1768 \varepsilon \operatorname{sech} \tau)-1, \\
\dot{x}=\operatorname{sech} \tau[0.7071+0.125 \varepsilon \operatorname{sech} \tau(1-\operatorname{sech} \tau)]\left[ \pm \operatorname{sech} \tau-0.1768 \varepsilon\left(2 \operatorname{sech}^{2} \tau-1\right)\right],
\end{gathered}
$$

where

$$
\tau=0.7071 t+0.2121 \arctan [\sinh (0.7071 t)]-0.2121 \tanh (0.7071 t)
$$

The solution by Cao's method can be derived as below,

$$
\begin{gathered}
x=-1+\cos \varphi, \\
\frac{d x}{d \varphi}=-\sin \varphi . \\
\frac{d \varphi}{d t}=( \pm 0.7071+0.125 \varepsilon) \sin \varphi .
\end{gathered}
$$

With $\varepsilon=1.2$, the phase portraits and time history diagrams of the solutions by different methods are shown in Figs. 9-11. The Runge-Kutta numerical solution at $\mu_{\mathrm{c}}$ is also shown for comparison. Here, the critical value $\mu_{\mathrm{c}}=0.5000$ is obtained, which is in agreement with that obtained by the present method. 


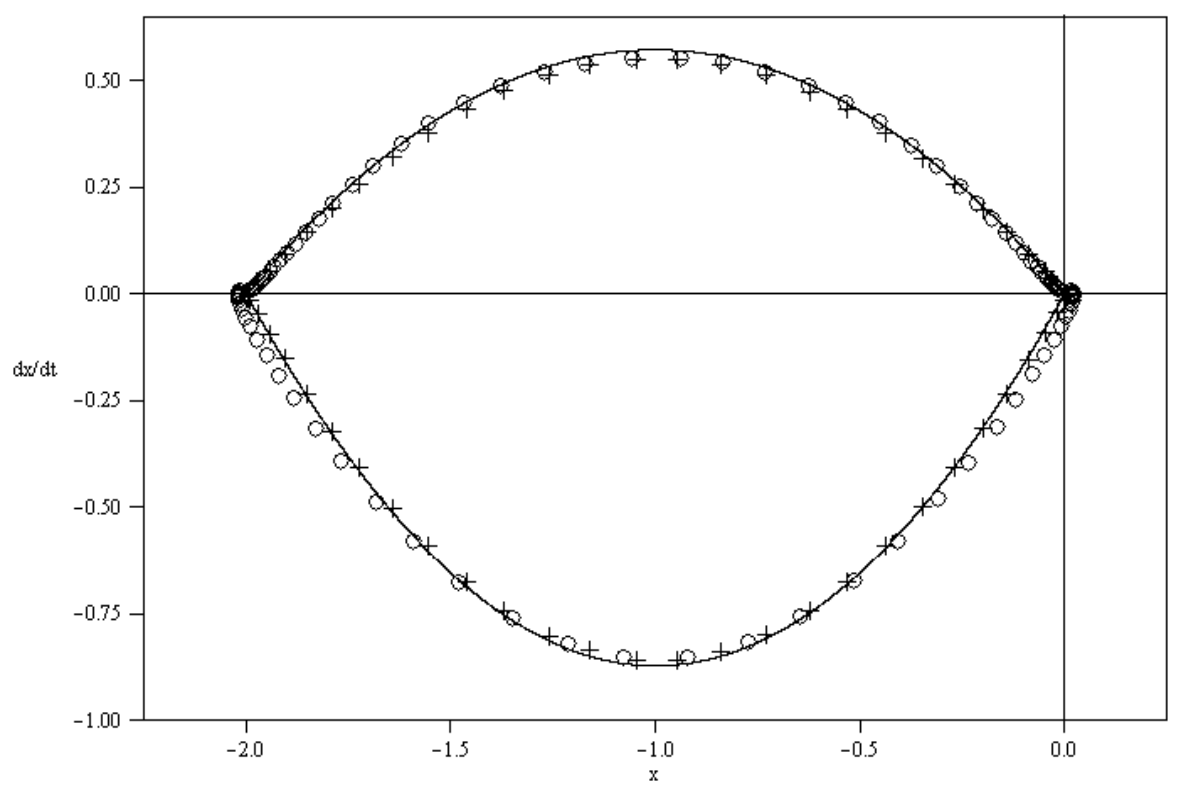

Figure 9. Heteroclinic orbits in phase portrait of Eq. (66). $\circ 0 \circ$ denotes the result by the present method; +++ denotes the result by Cao's method; - denotes the numerical orbits at $\mu_{\mathrm{c}}$ predicted by the Runge-Kutta method.

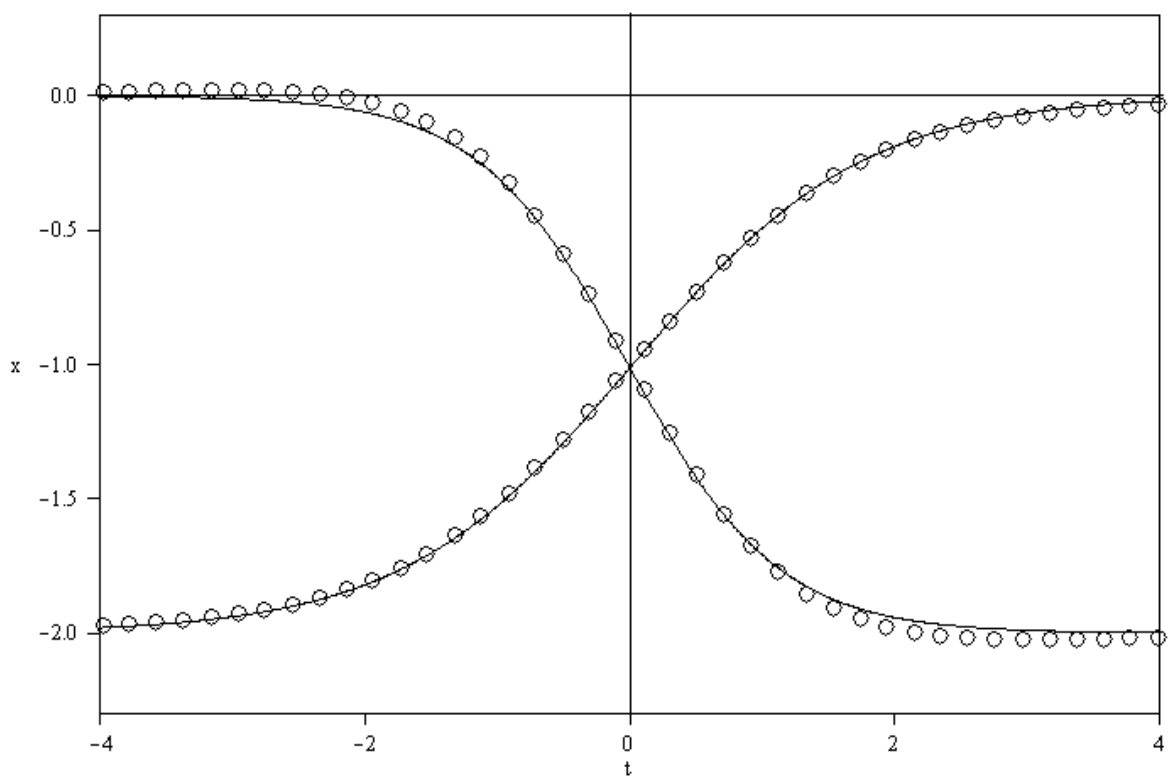

Figure 10. Amplitude history of heteroclinic solutions of Eq. (66). $० \circ \circ$ denotes the result by the present method; — denotes the numerical result at $\mu_{\mathrm{c}}$ by the Runge-Kutta method. 


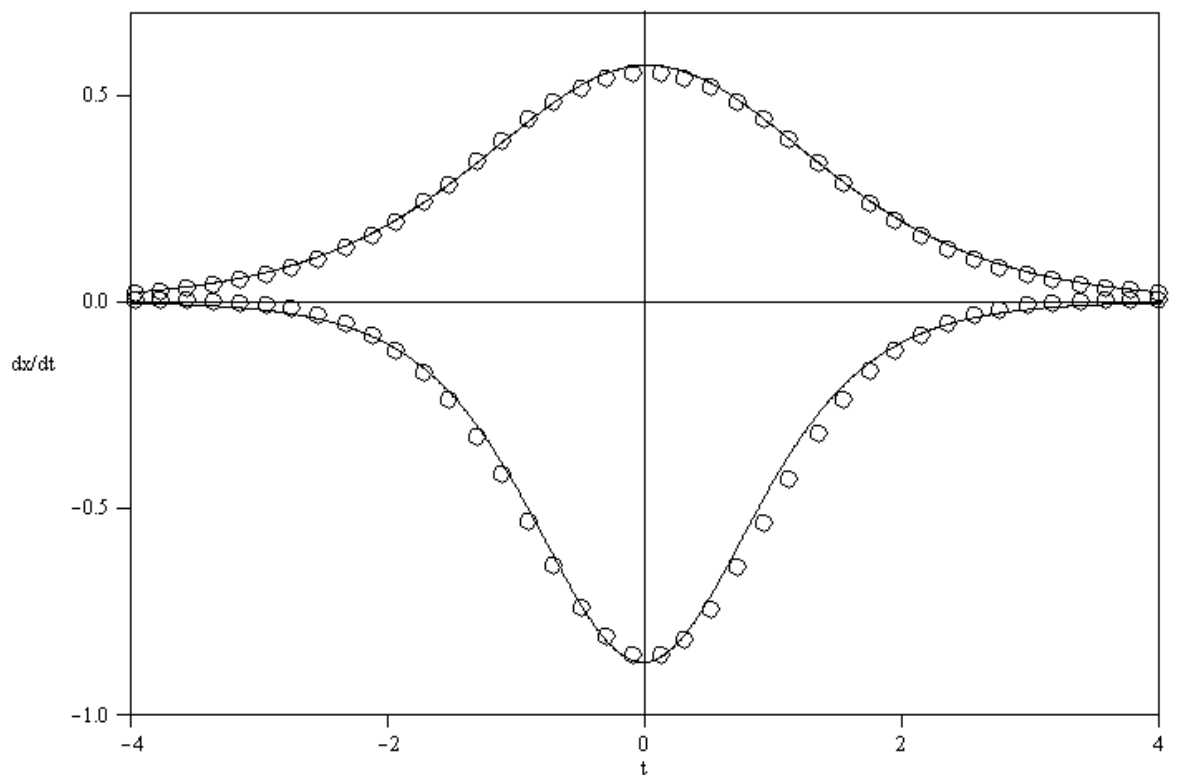

Figure 11. Velocity history of heteroclinic solutions of Eq. (66). $০ \circ \circ$ denotes the result by the present method; — denotes the numerical result at $\mu_{\mathrm{c}}$ by the Runge-Kutta method.

\section{Application to strongly quintic-septic nonlinearities}

\subsection{The perturbation procedure}

As an application of the present method, the following system is studied:

$$
\ddot{x}+c_{1} x+c_{5} x^{5}+c_{7} x^{7}=\varepsilon\left(\mu+\mu_{2} x^{2}\right) \dot{x}
$$

In other words,

$$
\begin{gathered}
g\left(x_{0}\right)=c_{1} x_{0}+c_{5} x_{0}^{5}+c_{7} x_{0}^{7}, \\
V\left(x_{0}\right)=\frac{1}{2} c_{1} x_{0}^{2}+\frac{1}{6} c_{5} x_{0}^{6}+\frac{1}{8} c_{7} x_{0}^{8}, \\
f\left(\mu, x_{0}, \omega_{0} x_{0}^{\prime}\right)=\omega_{0} x_{0}^{\prime}\left(\mu+\mu_{2} x_{0}^{2}\right),
\end{gathered}
$$

in which $\mu_{2}$ is constant. By substituting Eqs. (78) and (79) into Eqs. (5) and (6), respectively, one has

$$
\begin{gathered}
c_{1} b+c_{5} b^{5}+c_{7} b^{7}=0, c_{1}+5 c_{5} b^{4}+7 c_{7} b^{6} \leq 0 \\
\frac{1}{2} c_{1}\left(-a_{0}+b\right)^{2}+\frac{1}{6} c_{5}\left(-a_{0}+b\right)^{6}+\frac{1}{8} c_{7}\left(-a_{0}+b\right)^{8}=\frac{1}{2} c_{1}\left(a_{0}+b\right)^{2}+\frac{1}{6} c_{5}\left(a_{0}+b\right)^{6}+\frac{1}{8} c_{7}\left(a_{0}+b\right)^{8}
\end{gathered}
$$

by which $a_{0}$ and $b$ can be determined. Eq. (27) can be rewritten as

$$
\begin{gathered}
I(\tau)=\int_{0}^{\tau} x_{0}^{\prime} f\left(\mu, x_{0}, \sqrt{2\left[V\left(a_{0}+b\right)-V\left(x_{0}\right)\right]}\right) d \tau \\
=\sqrt{2} a_{0} \int_{0}^{\tau} \operatorname{sech}^{2} \tau\left[\mu+\mu_{2}\left(a_{0} \tanh \tau+b\right)^{2}\right] \sqrt{\left[V\left(a_{0}+b\right)-V\left(a_{0} \tanh \tau+b\right)\right]} d \tau .
\end{gathered}
$$


Thus by substituting Eqs. (79) and (82) into Eqs (29), (30) and (31), one can complete the calculation of the perturbation solution governed by Eqs. (32) and (33).

\subsection{Examples}

Two examples are presented in this section, for which Cao's method (Cao et al., 2011) are also applied. As Chen’s method (Chen et al., 2010) is only available for Duffing-type oscillator, it is ignored in this section. According to the discussion for Eqs. (34) and (35) in Section 3, as $\omega_{0}$ will not be derived as constants in the following examples, the present method, as well as Cao's method, are all implicit method in respect to $t$, and thus are fail to figure out the time history diagrams of the solutions.

Example 4. Consider the following oscillator:

$$
\ddot{x}+2 x-x^{5}-x^{7}=\varepsilon\left(\mu+x^{2}\right) \dot{x},
$$

which is a case of Eq. (36) with $c_{1}=2, c_{5}=-1, c_{7}=-1$ and $\mu_{2}=1$. From Eq. (40), $a_{0}= \pm 1$ and $b=0$. From Eq. (16),

$$
\omega_{0}(\tau)=\sqrt{\frac{5}{2}-\frac{4}{3} \operatorname{sech}^{2} \tau+\frac{1}{4} \operatorname{sech}^{4} \tau}=0.2887 \sqrt{30 \operatorname{sech}^{4} \tau-16 \operatorname{sech}^{2} \tau+3} .
$$

It should be noted that

$$
\operatorname{sech}(\tau)=\cos (\operatorname{am}(1, \tau)), \frac{d}{d \tau} \operatorname{am}(1, \tau)=\operatorname{sech}(\tau)
$$

where $\operatorname{am}(1, \tau)$ is the Jacobi amplitude function with the modulus 1 , and the approximate Fourier series expansion

$$
\sqrt{30 \operatorname{sech}^{4} \tau-16 \operatorname{sech}^{2} \tau+3}=P_{0}+P_{2} \cos (2 \mathrm{am}(\tau, 1))+P_{4} \cos (4 \mathrm{am}(\tau, 1)),
$$

in which

$$
P_{0}=0.4537, P_{2}=0.4199, P_{4}=-0.0330 \text {. }
$$

One can rewrite Eq. (82) as

$$
\begin{aligned}
I(\tau)= & \sqrt{2} a_{0} \int_{0}^{\tau} \cos (\operatorname{am}(\tau, 1))\left[\mu+0.5 \mu_{2} a_{0}^{2}(1-\cos (2 \operatorname{am}(\tau, 1)))\right]\left[P_{0}+P_{2} \cos (2 \operatorname{am}(\tau, 1))\right. \\
& \left.+P_{4} \cos (4 \operatorname{am}(\tau, 1))\right] d \operatorname{am}(\tau, 1)=\frac{\sqrt{2}}{105} a_{0} \tanh \tau\left(A_{1}+A_{2} \operatorname{sech}^{2} \tau+A_{4} \operatorname{sech}^{4} \tau+A_{6} \operatorname{sech}^{6} \tau\right),
\end{aligned}
$$

where

$$
\begin{gathered}
A_{1}=\mu\left(105 P_{0}+35 P_{2}-7 P_{4}\right)+\mu_{2} a_{0}^{2}\left(35 P_{0}-7 P_{2}-13 P_{4}\right), \\
A_{2}=14 \mu\left(5 P_{2}-4 P_{4}\right)-\mu_{2} a_{0}^{2}\left(35 P_{0}-49 P_{2}+59 P_{4}\right),
\end{gathered}
$$




$$
\begin{gathered}
A_{3}=168 \mu P_{4}+6 \mu_{2} a_{0}^{2}\left(32 P_{4}-7 P_{2}\right), \\
A_{4}=120 \mu_{2} a_{0}^{2} P_{4} .
\end{gathered}
$$

Then, substituting Eq. (88) into Eq. (29) gives

$$
A_{1}=0 \text {, }
$$

from which one derives

$$
\mu_{\mathrm{c}}=\frac{\mu_{2} a_{0}^{2}\left(-35 P_{0}+7 P_{2}+13 P_{4}\right)}{\left(105 P_{0}+35 P_{2}-7 P_{4}\right)}=-0.2137
$$

Substituting Eqs. (88) and (93) into Eq. (29) yields

$$
a_{1}=0
$$

Substituting Eqs. (88), (93) and (95) into Eq. (29) yields

$$
\omega_{1}=\frac{I(\tau)}{\omega_{0} x_{0}^{\prime 2}}=\frac{2 \sqrt{6}}{105} \frac{\tanh \tau}{\sqrt{30 \operatorname{sech}^{4} \tau-16 \operatorname{sech}^{2} \tau+3}}\left(\frac{A_{2}}{\operatorname{sech}^{2} \tau}+A_{4}+A_{6} \operatorname{sech}^{2} \tau\right) .
$$

Thus the heteroclinic solution of Eq. (83) is solved as

$$
\begin{gathered}
x= \pm \tanh \tau, \\
\dot{x}= \pm \operatorname{sech}^{2} \tau\left(\omega_{0}+\varepsilon \omega_{1}\right),
\end{gathered}
$$

where $\omega_{0}$ and $\omega_{1}$ are given in Eqs. (84) and (96).

The solution by Cao's method can be derived as below,

$$
\begin{gathered}
x=\cos \varphi, \\
\frac{d x}{d \varphi}=-\sin \varphi . \\
\frac{d \varphi}{d t}=\Phi_{0}(\varphi)+\varepsilon \Phi_{1}(\varphi) \\
\Phi_{0}(\varphi)= \pm 0.1667 \sin \varphi(8.2876+1.1746 \cos 2 \varphi+0.0264 \cos 4 \varphi) . \\
\Phi_{1}(\varphi)=(0.0343 \cos \varphi-0.0502 \cos 3 \varphi+0.0150 \cos 5 \varphi+0.0008 \cos 7 \varphi+ \\
0.0000 \cos 9 \varphi) /(1.3813+0.1958 \cos 2 \varphi+0.0044 \cos 4 \varphi) \sin 3 \varphi .
\end{gathered}
$$

The heteroclinic orbits with $\varepsilon=2$ are shown in Fig. 12. Similarly, the numerical phase portrait at $\mu_{\mathrm{c}}$ by the Runge-Kutta method is also shown for comparison. Here, the critical values $\mu_{\mathrm{c}}=-0.2138$, which is very close to that obtained by the present method. 


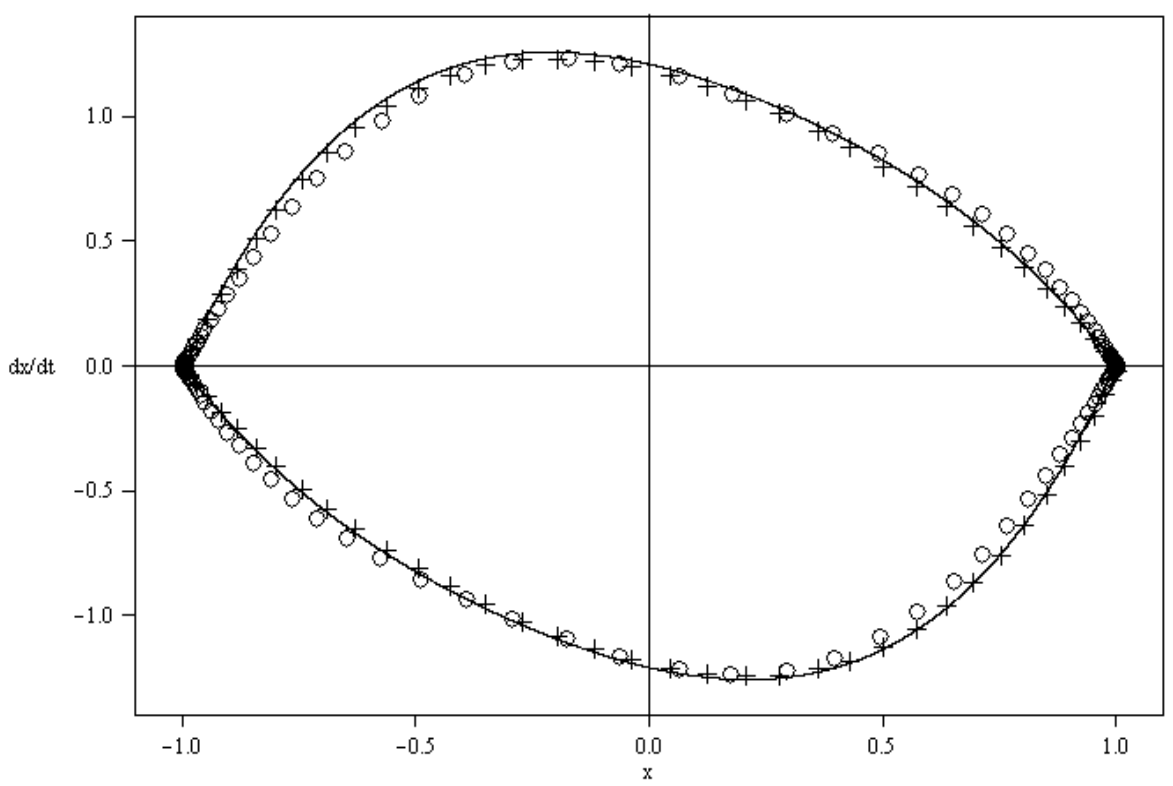

Figure 12. Heteroclinic orbits in phase portrait of Eq. (83). 000 denotes the result by the present method; +++ denotes the result by Cao's method; — denotes the numerical orbits at $\mu$ c predicted by the Runge-Kutta method.

Example 5. Consider the following oscillator:

$$
\ddot{x}+x+x^{5}-x^{7}=\varepsilon\left(\mu-x^{2}\right) \dot{x},
$$

which is a case of Eq. (36) with $c_{1}=1, c_{5}=1, c_{7}=-1$ and $\mu_{2}=-1$. From Eq. (40), $a_{0}= \pm 1.211$ and $b=$ 0. From Eq. (16),

$$
\omega_{0}(\tau)=\sqrt{0.7870 \operatorname{sech}^{4} \tau-2.4320 \operatorname{sech}^{2} \tau+2.5739}
$$

Noting Eq. (85 and using the approximate Fourier series expansion, one can have

$$
\begin{aligned}
& \operatorname{sech}^{2} \tau \sqrt{0.5767 \operatorname{sech}^{4} \tau-1.7821 \operatorname{sech}^{2} \tau+1.8862} \\
& =P_{0}+P_{2} \cos (2 \operatorname{am}(\tau, 1))+P_{4} \cos (4 \operatorname{am}(\tau, 1))
\end{aligned}
$$

in which

$$
P_{0}=0.4725, P_{2}=0.4074, P_{4}=-0.0606
$$

Then, one can follow the same procedure as expressed by Eqs. (88)-(93) and substitute Eqs. (107) into Eq. (94) to get

$$
\mu_{\mathrm{c}}=\frac{\mu_{2} a_{0}^{2}\left(-35 P_{0}+7 P_{2}+13 P_{4}\right)}{\left(105 P_{0}+35 P_{2}-7 P_{4}\right)}=0.3299 .
$$


Substituting Eqs. (88) and (93) into Eq. (29) yields

$$
a_{1}=0 .
$$

Substituting Eqs. (88), (93) and (109) into Eq. (29) yields

$$
\omega_{1}=\frac{I(\tau)}{\omega_{0} x_{0}^{\prime 2}}=\frac{2 \sqrt{6}}{105} \frac{\tanh \tau}{\sqrt{30 \operatorname{sech}^{4} \tau-16 \operatorname{sech}^{2} \tau+3}}\left(\frac{A_{2}}{\operatorname{sech}^{2} \tau}+A_{4}+A_{6} \operatorname{sech}^{2} \tau\right) .
$$

Thus the heteroclinic solution of Eq. (83) is solved as

$$
\begin{gathered}
x= \pm \tanh \tau, \\
\dot{x}= \pm \operatorname{sech}^{2} \tau\left(\omega_{0}+\varepsilon \omega_{1}\right),
\end{gathered}
$$

where $\omega_{0}$ and $\omega_{1}$ are given in Eqs. (84) and (110).

The solution by Cao's method can be derived as below,

$$
\begin{gathered}
x=\cos \varphi, \\
\frac{d x}{d \varphi}=-\sin \varphi, \\
\frac{d \varphi}{d t}=\Phi_{0}(\varphi)+\varepsilon \Phi_{1}(\varphi), \\
\Phi_{0}(\varphi)= \pm(0.5520-0.4759 \cos 2 \varphi-0.0708 \cos 4 \varphi) / \sin \varphi, \\
\Phi_{1}(\varphi)=(-0.0454 \cos \varphi+0.0650 \cos 3 \varphi-0.0177 \cos 5 \varphi- \\
0.0019 \cos 7 \varphi /(0.5520-0.4759 \cos 2 \varphi-0.0708 \cos 4 \varphi) \sin \varphi .
\end{gathered}
$$

The heteroclinic orbits with $\varepsilon=1.5$ are shown in Fig. 13. Similarly, the numerical phase portrait at $\mu_{\mathrm{c}}$ by the Runge-Kutta method is also shown for comparison. Here, the critical values $\mu_{\mathrm{c}}=0.3311$, which is very close to that obtained by the present method. 


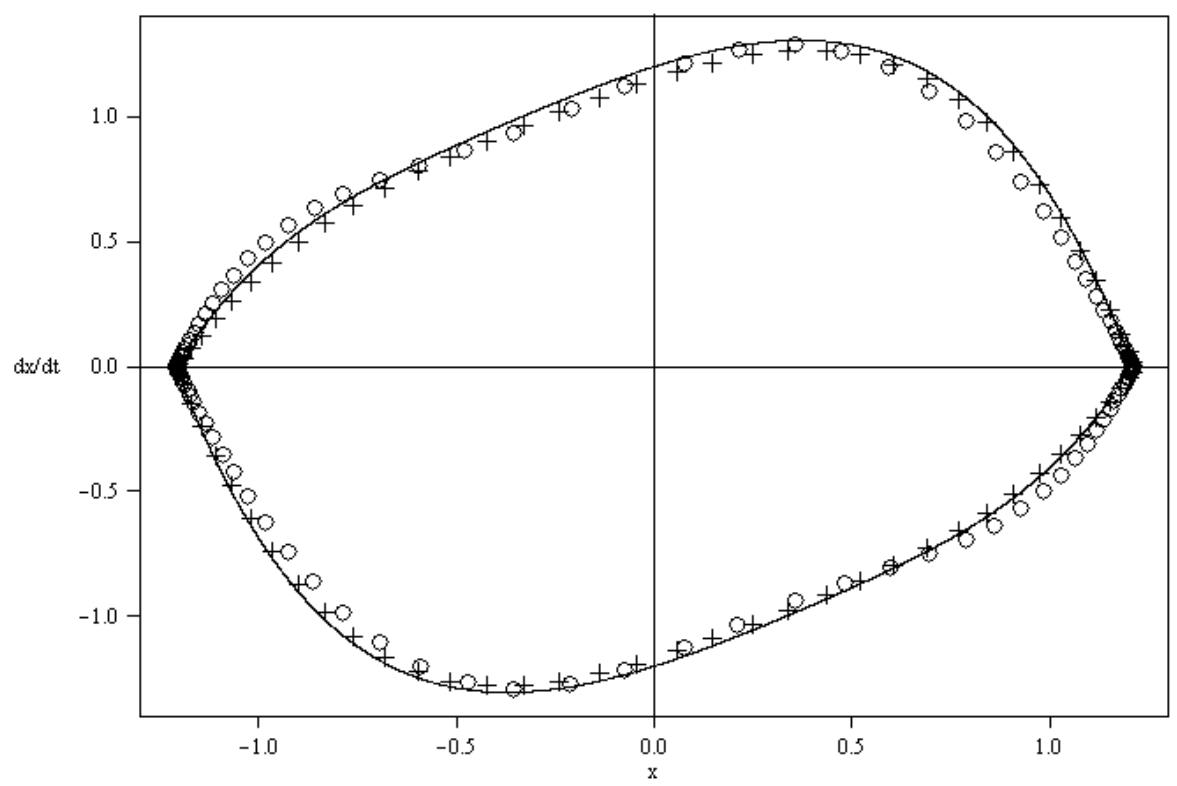

Figure 13. Heteroclinic orbits in phase portrait of Eq. (104). 000 denotes the result by the present method; ++ + denotes the result by Cao's method; - denotes the numerical orbits at $\mu_{\mathrm{c}}$ predicted by the Runge-Kutta method.

\section{Conclusions}

(1) The generalized hyperbolic perturbation method presented is an effective method for determining heteroclinic solutions of certain nonlinear oscillators in the form of $\ddot{x}+g(x)=\varepsilon f(\mu, x, \dot{x})$. The previous hyperbolic perturbation solutions for Duffing type oscillator (Chen and Chen, 2009) can be just regarded as a special case of the present method.

(2) Based on the generalized hyperbolic functions and the nonlinear time transformation proposed, the generalized heteroclinic solutions are defined and adopted as the basic functions in the perturbation procedures. The critical values of the heteroclinic bifurcation parameter are also obtained in the procedure. The reliable accuracy of the proposed method is demonstrated by solving strongly nonlinear oscillators with quadratic-cubic nonlinearities and with quintic-septic nonlinearities.

(3) The present method shows a higher accuracy and efficiency than those by Chen's hyperbolic Lindstedt-Poincaré method. It is recommended to use the present method to seek explicit heteroclinic solutions in the infinite time domain for some typical system such as Helmholtz-Duffing (quadratic-cubic) oscillators, and to use Cao's perturbation-incremental method to construct implicit and semi-analytical heteroclinic orbits in phase portrait for strongly nonlinear systems with arbitrary 
large $\varepsilon$. It is still desirable to develop new techniques to analytically construct the solutions explicitly in respect to original time, for systems with complex nonlinearities.

\section{Acknowledgment}

The financial supports from the National Natural Science Foundation of China (11102045), the National Basic Research Program of China (2011CB013606) and the National Plan for Science and Technology Support of China (2012BAJ07B02) are gratefully acknowledged.

\section{Appendix}

To illustrate a typical occurrence of the heteroclinic connection, and the evolution of a limit cycle under parameter control, a series of phase portraits for a self-excited system governed by Eq.(104) is shown in Figs. 14-19. In the figures, the black trajectories, signed with time direction arrows, stand for the stable and unstable manifolds of the saddle points. They divide the phase portrait into different areas.

Fig. 14 shows a simple conservative phase portrait structure, in which a series of concentric periodic orbits colored in yellow are bounded in the heteroclinic orbit, while the green trajectories denote those unstable trajectories surrounded.

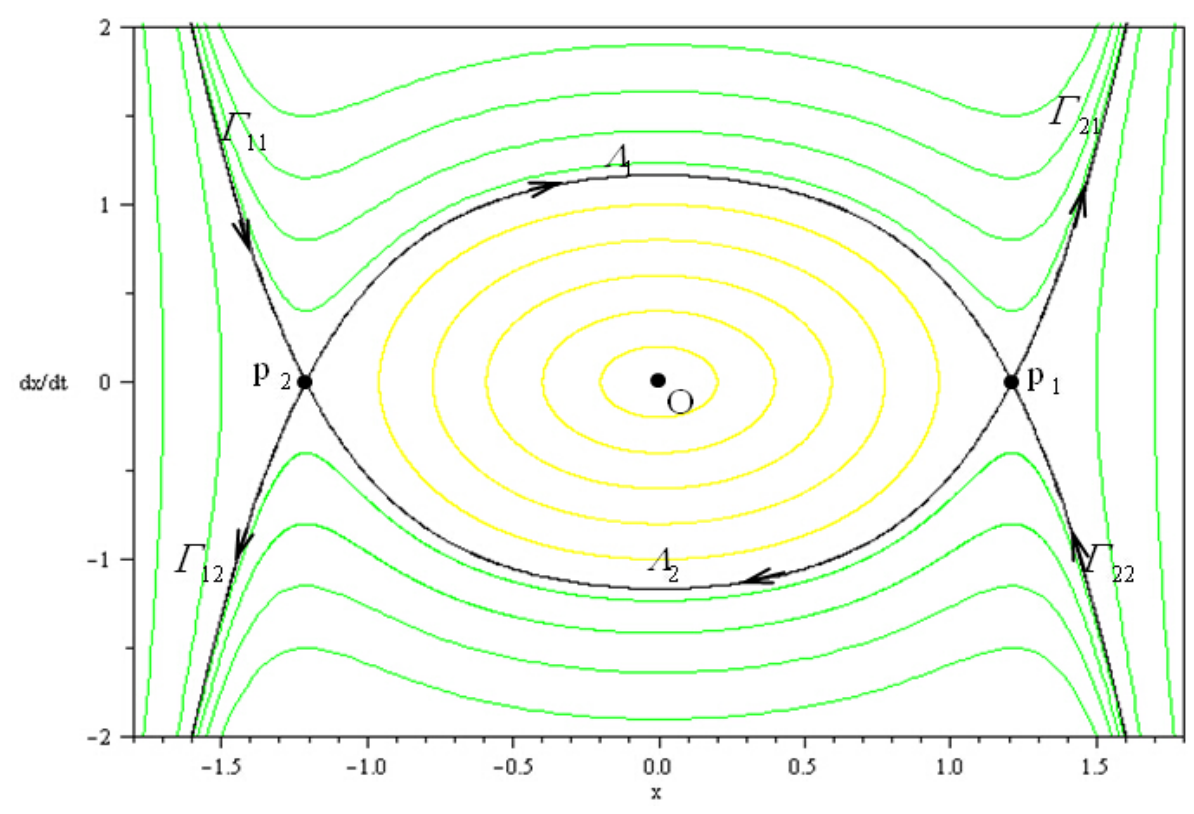

Figure 14. Phase portrait of Eq.(104) with $\varepsilon=0$ 
Fig. 15 shows the condition at a so-called Hopf bifurcation, in which the non-transverse intersections of the stable and unstable manifolds disappear and, the focus at point $\mathrm{O}$ possesses a so-called attraction basin, which is bounded by the stable manifolds $\Gamma_{11}, \Gamma_{13}, \Gamma_{22}$, and $\Gamma_{24}$. It can be seen that the separated manifolds $\Gamma_{14}, \Gamma_{23}, \Gamma_{14}$, and $\Gamma_{23}$, divide the attraction basin into two areas, in which the flows are colored in blue and red, respectively.

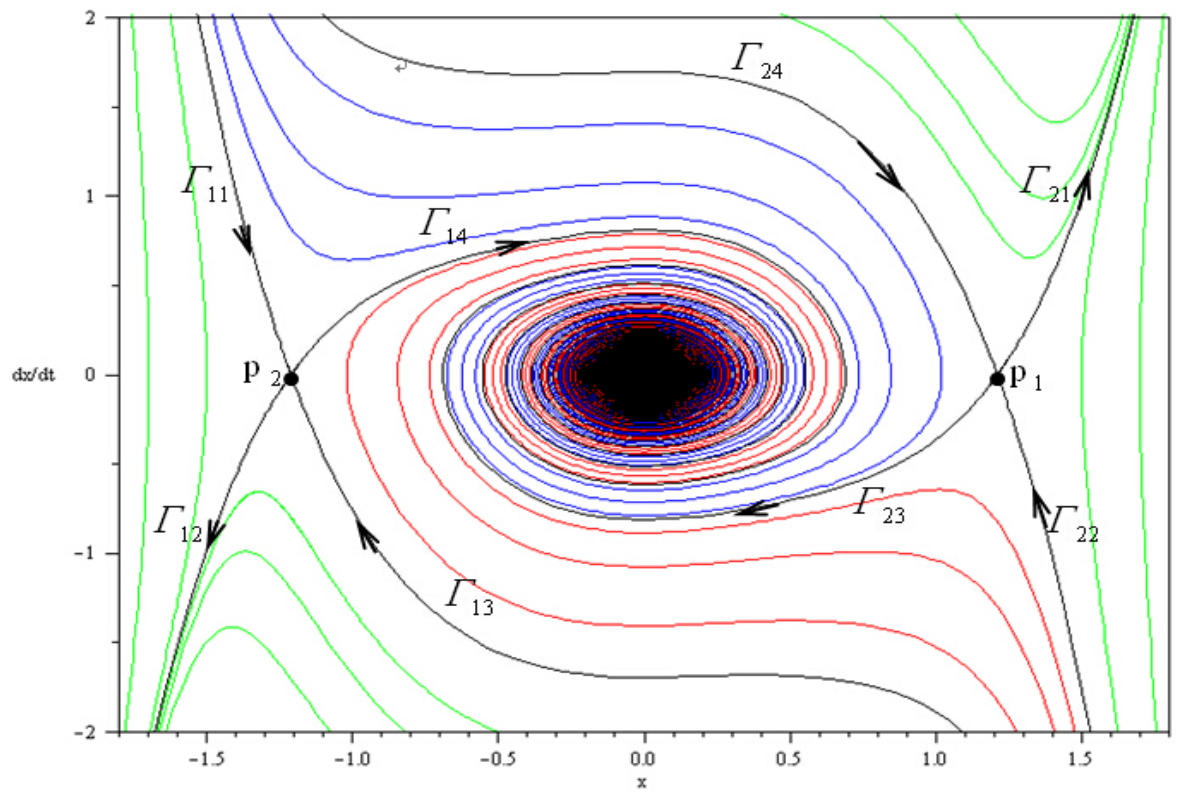

Figure 15. Phase portrait of Eq.(104) with $\varepsilon=1.5, \mu=0$

Fig. 16 presents a limit cycle, with its attraction basin bounded by the stable manifolds $\Gamma_{11}, \Gamma_{13}$, $\Gamma_{22}$, and $\Gamma_{24}$, emergences slightly after the Hopf bifurcation.

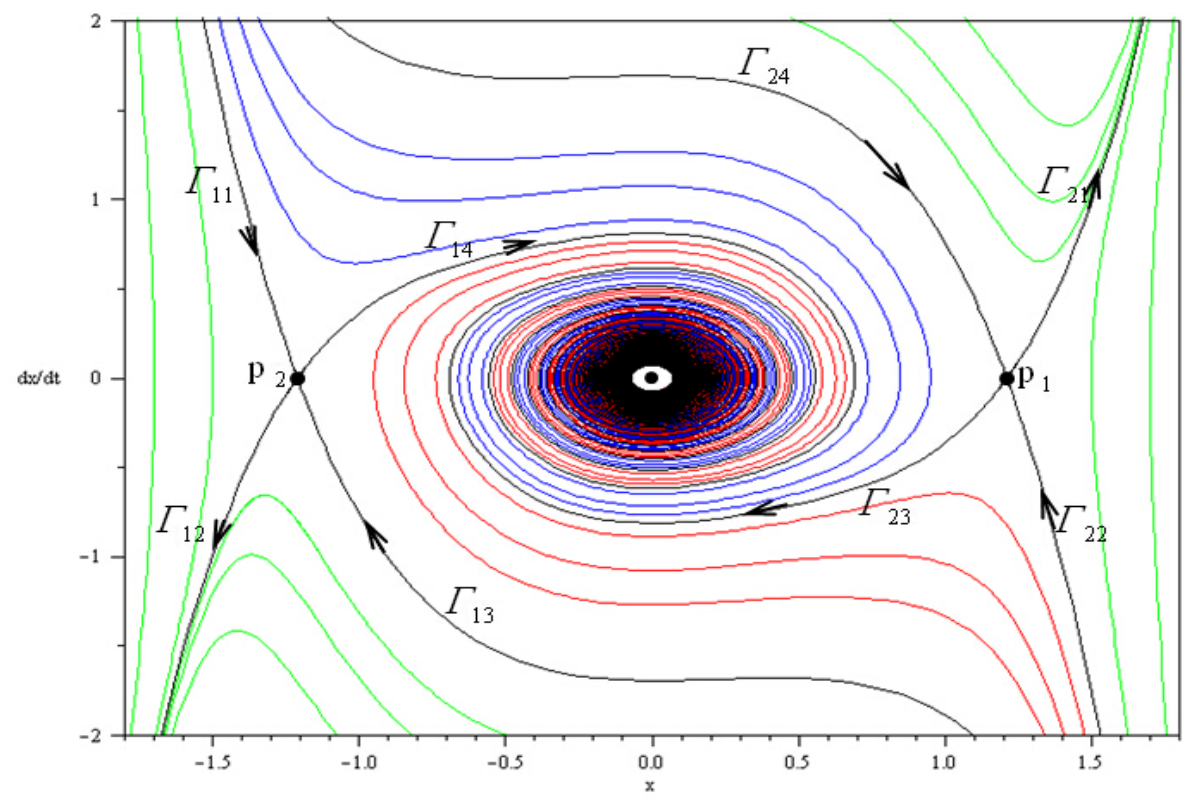

Figure 16. Phase portrait of Eq.(104) with $\varepsilon=1.5, \mu=0.001$ 
Fig. 17 shows the condition under which the limit cycle is becoming larger and, the stable and unstable manifolds, $\Gamma_{13}$ and $\Gamma_{23}, \Gamma_{14}$ and $\Gamma_{24}$, are getting closer to non-transverse intersection.

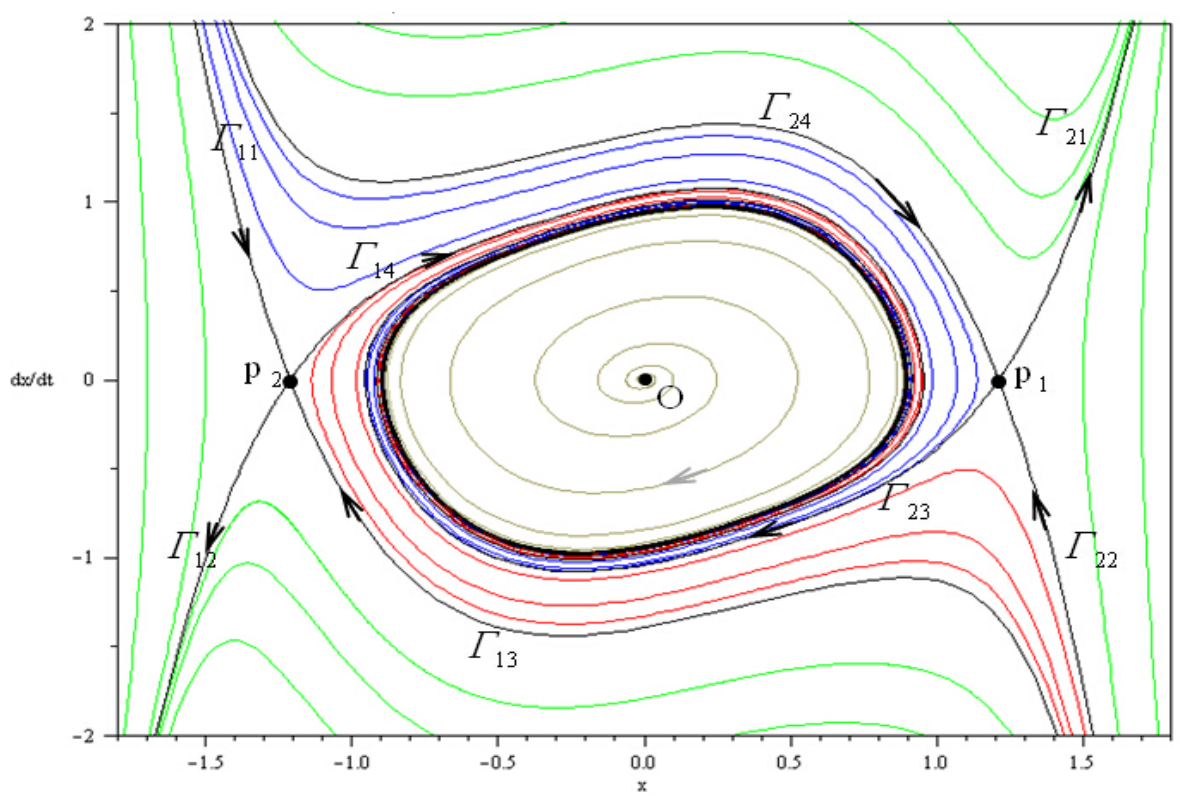

Figure 17. Phase portrait of Eq.(104) with $\varepsilon=1.5, \mu=0.2$

Fig.18 presents the condition at the heteroclinic bifurcation, in which the limit cycle breaks, and the half-heteroclinic orbit $\Lambda_{1}$ has been formed by the non-transverse intersection of manifolds $\Gamma_{13}$ and $\Gamma_{23}$. The other half-heteroclinic orbit $\Lambda_{2}$ has been formed by non-transverse intersection of manifolds by $\Gamma_{14}$ and $\Gamma_{24}$. It can be seen clearly that the heteroclinic orbit is the boundary of the maximum vibration amplitude of the limit cycle evolution.

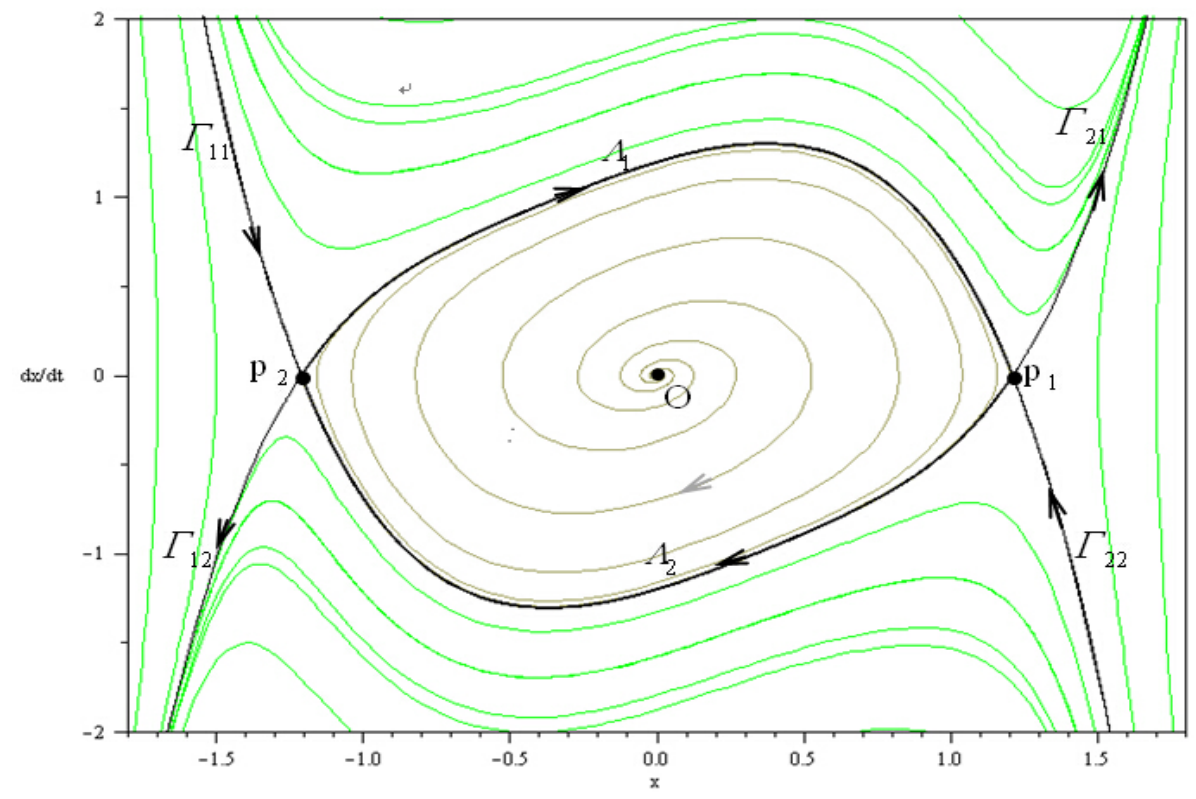

Figure 18. Phase portrait of Eq.(104) with $\varepsilon=1.5, \mu=0.331101$ 
Fig. 19 shows a condition after the heteroclinic bifurcation.

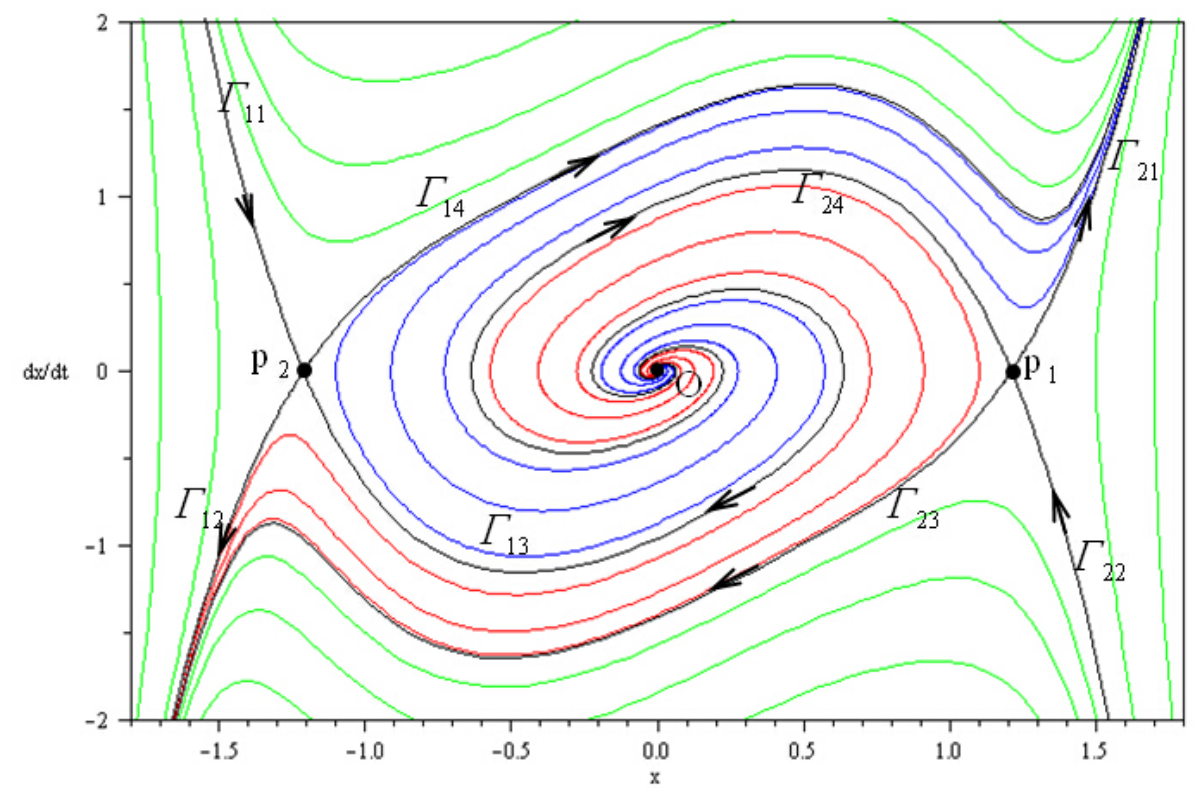

Figure 19. Phase portrait of Eq.(104) with $\varepsilon=1.5, \mu=0.5$

\section{References}

Belhaq M, Fiedler B and Lakrad F (2000) Homoclinic connections in strongly self-excited nonlinear oscillators: the Melnikov function and the elliptic Lindstedt-Poincaré method. Nonlinear Dynamics. 23, 67-86.

Belhaq M and Lakrad F (2000) Prediction of homoclinic bifurcation: the elliptic averaging method. Chaos Solitons \& Fractals 11: 2251-2258.

Cao H, Jiang Y and Shan Y (2006) Primary resonant optimal control for nested homoclinic and heteroclinic bifurcation in single-dof nonlinear oscillators. Journal of Sound and Vibration 289: 229-244.

Cao YY, Chung KW and Xu J (2011) A novel construction of homoclinic and heteroclinic orbits in nonlinear oscillators by a perturbation-incremental method. Nonlinear Dynamics 64: 221-236.

Chan HSY, Chung KW and Xu Z (1997) Stability and bifurcations of limit cycles by the perturbation-incremental method. Journal of Sound and Vibration 206: 589-604.

Chen SH, Chen YY and Sze KY (2010) Homoclinic and heteroclinic solutions of cubic strongly nonlinear autonomous oscillators by hyperbolic Lindstedt-Poincaré method. Science China: Technological Sciences 53: 692-702.

Chen YY and Chen SH (2009) Homoclinic and heteroclinic solutions of cubic strongly nonlinear autonomous oscillators by the hyperbolic perturbation method. Nonlinear Dynamics 58: 417-429. 
Chen YY, Chen SH and Sze KY (2009) A hyperbolic Lindstedt-Poincaré method for homoclinic motion of a kind of strongly nonlinear autonomous oscillators. Acta Mechanica Sinica 25: 721-729.

Chen YY, Yan LW, Sze KY and Chen SH (2012) Generalized hyperbolic perturbation method for homoclinic solutions of strongly nonlinear autonomous systems. Applied Mathematics and Mechanics 33: 1137-1152.

Feng JJ, Zhang QC and Wang W (2012) Chaos of several typical asymmetric systems. Chaos Solitons \& Fractals 45: 950-958.

Guckenheimer J and Holmes P (2002) Nonlinear Oscillations, Dynamical Systems, and Bifurcations of Vector Fields. New York: Springer Verlag.

Hale JK, Peletier LA and Troy WC (2000) Exact homoclinic and heteroclinic solutions of the Gray-Scott model for autocatalysis. SIAM Journal on Applied Mathematics 61: 102-130.

Lenci S and Rega G (2003) Optimal control of homoclinic bifurcation: theoretical treatment and practical reduction of safe basin erosion in the Helmholtz oscillator. Journal of Vibration and Control 9: 281-315.

Li ZB, Tang JS and Cai P (2013) A generalized harmonic function perturbation method for determining limit cycles and homoclinic orbits of Helmholtz-Duffing oscillator. Journal of Sound and Vibration 332: 5508-5522.

Manucharyan GV and Mikhlin YV (2005) The construction of homo- and heteroclinic orbits in non-linear systems. Journal of Applied Mathematics and Mechanics 69: 42-52.

Melnikov VK (1963) On the stability of the center for time periodic perturbations. Transaction of Moscow Mathematical Society 12: 1-57.

Merkin JH and Needham DJ (1986) On Infinite Period Bifurcations with an Application to Roll Waves. Acta Mechanica 60: 1-16.

Mikhlin YV and Manucharyan GV (2003) Construction of homoclinic and heteroclinic trajectories in mechanical systems with several equilibrium positions. Chaos, Solitons \& Fractals. 16, 299-309.

Nayfeh AH and Balachandran B (1995) Applied Nonlinear Dynamics: Analytical, Computational, and Experimental Method. New York: Wiley-Interscience.

Nayfeh AH and Pai PF (2004) Linear and Nonlinear Structural Mechanics. New York: Wiley-Interscience.

Rega G and Lenci S (2008) Dynamical integrity and control of nonlinear mechanical oscillators. Journal of Vibration and Control 14: 159-179.

Uzunov IM (2010) Description of the suppression of the soliton self-frequency shift by bandwidth-limited amplification. Physical Review E 82: 066603 1-8. 
Uzunov IM and Arabadzhiev TN (2011) Suppression of the soliton self-frequency shift and compression in the presence of bandwidth-limited amplification. Physical Review E 84: 026607 $1-9$.

Xu Z, Chan HSY and Chung KW (1996) Separatrices and limit cycles of strongly nonlinear oscillators by the perturbation-incremental method. Nonlinear Dynamics 11: 213-233.

Zhang QC, Wang W and Li WY (2008) Heteroclinic bifurcations of strongly nonlinear oscillator. Chinese Physics Letters 25: 1905-1907. 